\title{
Examples of Lorenz-like Attractors in Hénon-like Maps
}

\author{
S.V. Gonchenko ${ }^{1}$, A.S. Gonchenko ${ }^{1}$, I.I. Ovsyannikov ${ }^{12}$, D.V. Turaev ${ }^{2}$ \\ ${ }^{1}$ Research Institute of Applied Mathematics and Cybernetics, \\ 10, Ulyanova Str. 603005 Nizhny Novgorod, Russia \\ ${ }^{2}$ Imperial College, SW7 2AZ London, UK
}

\begin{abstract}
We display a gallery of Lorenz-like attractors that emerge in a class of threedimensional maps. We review the theory of Lorenz-like attractors for diffeomorphisms (as opposed to flows), define various types of such attractors, and find sufficient conditions for three-dimensional Henon-like maps to possess pseudohyperbolic Lorenz-like attractors. The numerically obtained scenarios of the creation and destruction of these attractors are also presented.
\end{abstract}

Keywords and phrases: Strange attractor, three-dimensional maps, pseudohyperbolicity, volume hyperbolicity, bifurcations, homoclinic orbit, homoclinic tangency, wild set, normal form

Mathematics Subject Classification: 34D45, 37C70, 37D45

\section{Introduction}

Like the dynamics of three-dimensional systems of differential equations can be drastically different from that of two-dimensional systems, the dynamics of three-dimensional diffeomorphisms can be very much unlike the dynamics of two-dimensional ones. Chaotic behaviour can be observed both in two-dimensional and three-dimensional maps. However, especially when the Jacobian of a three-dimensional map is not too close to zero, the chaos can take forms which have nothing in common with what one observes in two-dimensional diffeomorphisms.

One of these phenomena, discovered in [1], is the emergence of Lorenz-like attractors for diffeomorphisms of dimension 3 and higher. We will give a precise definition of a Lorenz-like attractor for a diffeomorphism later (Section 2). Here, we just note that it has both fundamental differences and fundamental similarities with/to the Lorenz attractors for systems of differential equation (i.e. for flows). To distinguish between them we reserve the term "Lorenz attractor" for the case of flows and "Lorenz-like attractor" for the case of diffeomorphisms (see a terminological summary in the table in Section 2). The most important property of the classical Lorenz attractor for flows is that it is a genuine strange attractor: it contains no stable periodic orbits, and every orbit in such attractor has positive maximal Lyapunov exponent $[2,3]$. Moreover, these properties are robust (they persist at small changes of the right-hand sides), eventhough the attractor itself is structurally unstable [2-6]. We note that this property of the robust

${ }^{*}$ Corresponding author. E-mail: gonchenko@pochta.ru 
instability seemingly does not hold for many "physical" attractors that appear in numerical experiments, where an observed chaotic behavior can easily correspond to some periodic orbit with a very large period (plus inevitable noise); see more discussion in $[9,11]$. In particular, Hénon-like strange attractors [13-15] that are often in two-dimensional maps may transform into stable long-period orbits by arbitrarily small changes of parameters [16].

The reason for the robust chaoticity of the classical Lorenz attractor is that it possesses a pseudohyperbolic or, which is the same, a volume-hyperbolic structure, which we describe in detail in Section 2. Lorenz-like attractors for diffeomorphisms share the same fundamental property. As a result, they too do not contain stable periodic orbits, nor any stable orbit can emerge in a neighbourhood of the Lorenz-like attractor at small perturbations of the diffeomorphism. Thus, the fact that these attractors exist in an important class of three-dimensional maps is remarkable. It provides us with an example of truly high-dimensional (i.e. not two-dimensional) robust chaotic behaviour which must be frequent in various applications. In particular, a Lorenz-like attractor for a Poincare map of a model of celtic stone was found in [17].

As we mentioned, the dynamics of Lorenz-like attractors for diffeomorphisms is actually different from the dynamics of Lorenz attractors for flows (by taking the flow suspension of a three-dimensional diffeomorphism with a Lorenz-like attractor we obtain a four-dimensional flow whose attractor may contain Newhouse wild sets and saddle periodic orbits with three-dimensional unstable manifolds [19], as well as saddle invariant tori; none of these objects is present within the classical Lorenz attractor). However, visually, a Lorenz-like attractor of a diffeomorphism may look quite similar to the classical Lorenz attractor. Thus Fig. 1 (left) shows a picture of the attractor numerically obtained in [1] for the map

$$
\bar{x}=y, \quad \bar{y}=z, \quad \bar{z}=M_{1}+B x+M_{2} y-z^{2},
$$

at $M_{1}=0, M_{2}=0.85$ and $B=0.7$. In spite of the striking similarity to the attractor of the Lorenz model, this picture is not a discretisation of a continuous trajectory of a system of differential equations. In fact, the distance between two consecutive points on the orbit of map (1.1) shown in Fig. 1 does not stay small (this orbit lies close to the one-dimensional unstable separatrix of a fixed point whose unstable multiplier is negative; the fixed point divides the separatrix into two parts which are interchanged by the map, so each iteration lands to the opposite part of the separatrix). The reason for the similarity of the attractor of map (1.1) to a discretisaton of the Lorenz model is "genetic". In the space of parameters $\left(M_{1}, M_{2}, B\right)$ there is a path to the point $M_{1}=-\frac{1}{4}, M_{2}=1$ and $B=1$ such that the attractor, when we move along this path, shrinks to a fixed point $x=y=z=\frac{1}{2}$. This fixed point has multipliers $(-1,-1,1)$. According to [23], the normal form for the second iteration of the map near such point is close to the time-1 map of the Morioka-Shimizu model [20]. This system of three differential equations is known to have a Lorenz attractor for a region of parameter values [21-23], i.e. near this bifurcation point the second iteration of the map can be close, in some small neighbourhood of the fixed point, to a discretisation of a flow with a Lorenz attractor (after a proper scaling of the coordinates). The birth of a small Lorenz-like attractor at this bifurcation in map (1.1) was indeed established in [1]. As we move away from the bifurcation point in the parameter space, the attractor grows in size significantly. However, eventhough the parameter values corresponding to the attractors shown in Fig. 1 are quite far from the bifurcation moment, it is possible that there still exist some coordinates in which the second iteration of the map is not very far from the time-1 map of some flow.

In this paper we display Lorenz-like attractors that emerge in the class of three-dimensional Henon-like maps of the form

$$
\bar{x}=y, \quad \bar{y}=z, \quad \bar{z}=B x+f(y, z),
$$

where $f$ is a smooth function. The Jacobian of this map is constant and equals $B$, i.e. $B=1$ is the case of volume-preserving maps while small values $B$ correspond to the strong volume-contraction. We find our Lorenz attractors for values of $B<1$ that are still closer to the volume-preserving than to volume-contracting case. Map (1.1) is, obviously, a partial case of (1.2). 

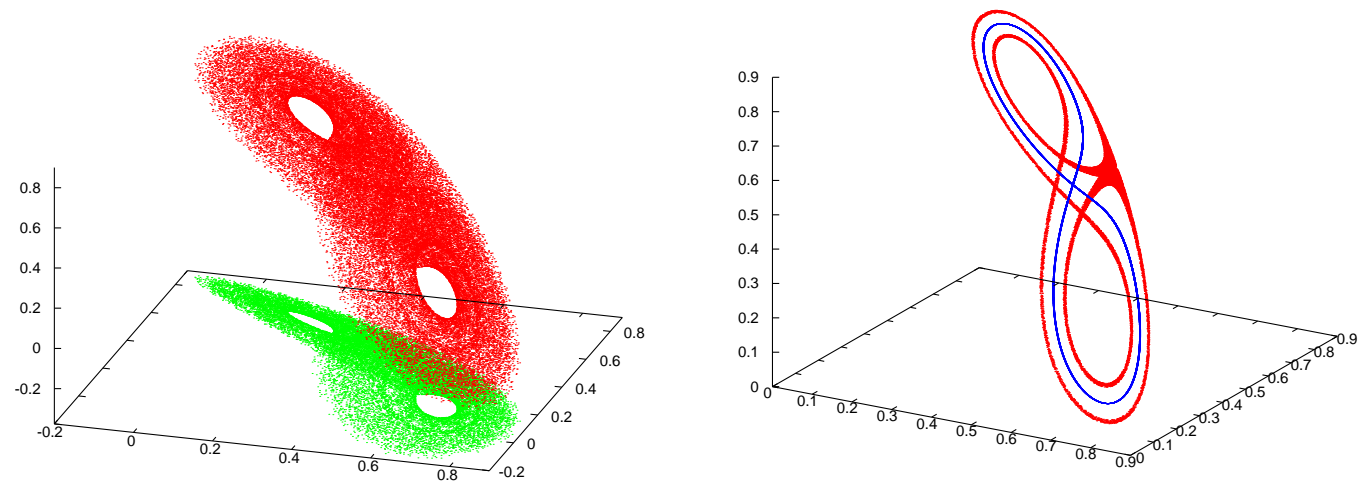

FiguRE 1. Plots of attractors of map (1.1) observed numerically in [1] for $M_{1}=0, B=0.7$ and $M_{2}=0.85$ (left) or $M_{2}=0.815$ (right). In the left panel, the projection on the $(x, y)$-plane is also displayed. In the right panel, a "figure-eight" saddle closed invariant curve inside the lacuna is shown. Note the similarity to the Lorenz attractors of the Shimizu-Morioka system (2.1), see [21,22].

The general interest to Henon-like maps (not necessarily three-dimensional) is due to several reasons. For example, it is well-known that maps of this type appear naturally in the study of groups of polynomial diffeomorphisms (i.e. polynomial maps with polynomial inverse), volume-preserving and symplectic polynomial diffeomorphisms [24-27,39, 80,81]. Most importantly, Henon-like maps emerge as rescaled first-return maps near various types of homoclinic orbits, see [28]. The first in the list of the maps of "homoclinic origin" is the parabola map

$$
\bar{x}=y, \quad \bar{y}=M-y^{2},
$$

It was shown in [29] that this map emerges as a limit model for the rescaled first-return map near a quadratic homoclinic tangency for area-contracting two-dimensional maps. The same map appears in the study of homoclinic tangencies in the so-called sectionally dissipative case (multi-dimensional maps that contract two-dimensional areas) [34,36,38]. In fact, parabola map (1.3) universally appears near all types of homoclinic tangencies, as an approximation of the restriction of first-return maps (or their inverse maps) to certain invariant manifolds $[35,37,42,48]$. It also emerges in the study of homoclinic loops to a saddle-saddle equilibrium [40].

The standard Hénon map

$$
\bar{x}=y, \quad \bar{y}=M-b x-y^{2}
$$

was explicitly introduced into the dynamical systems theory in [30]. It was also considered (with $b$ small) in relation to the study of homoclinic tangencies in [31]. In general, this map emerges as the limit model for first-return maps near homoclinic tangencies in the situations where two-dimensional areas are not contracted, while three-dimensional volumes are, see $[34,39,42,46,48,50]$. It also appears in the study of homoclinic tangencies in two-dimensional endomorphisms [55]. In terms of $[41,46,48]$, this map appears in problems of effective dimension 2. As the bifurcation (at $b=1$ ) of a pair of multipliers on the unit circle is degenerate in the standard Hénon map, the generalized Hénon map (GHM)

$$
\bar{x}=y, \quad \bar{y}=M-b x-y^{2}+\nu_{1} x y+\nu_{2} y^{3},
$$

was proposed in $[51,52]$ as a more accurate approximation to the first-return maps near homoclinic tangencies in the case of effective dimension 2 (a conservative analogue of GHM, i.e. the case $\nu_{1}=0$, was introduced in $[60,61])$. Here, the parameters $M$ and $b$ can take arbitrary values, while $\nu_{1,2}$ are small. 
The degeneracy of the Hénon map is canceled in GHM at nonzero $\nu_{1}$ (see [53]), so the analysis of this map provides an accurate information on the birth of closed invarant curves near homoclinic tangencies [46, 48,50-52,55], homoclinic loops [56], and heteroclinic cycles [54]. Note also that map (1.5) at $\nu_{1,2}$ not small demonstrates interesting hyperbolic dynamics (the so-called half-orientable horseshoes) [57-59].

The conservative Hénon map

$$
\bar{x}=y, \quad \bar{y}=M-x-y^{2},
$$

and the conservative GHM

$$
\bar{x}=y, \quad \bar{y}=M-x-y^{2}+\nu y^{3},
$$

appear in the study of area-preserving and/or reversible maps with quadratic homoclinic tangencies [6265,68-74], volume-preserving flows with homoclinic loops to a saddle-focus [60,61], billiard-like potentials [66,67]. A four-dimensional symplectic Hénon map

$$
\begin{aligned}
& \bar{x}_{2}=x_{1}, \quad \bar{x}_{1}=y_{2}, \quad \bar{y}_{2}=y_{1}, \\
& \bar{y}_{1}=M_{1}\left(x_{1}+y_{1}\right)-x_{2}-y_{2}^{2}+M_{2}
\end{aligned}
$$

was obtained in $[75,76]$ as a rescaled limit of the first-return map near a homoclinic tangency for a saddle-focus periodic orbit.

The Mira map

$$
\bar{x}=y, \quad \bar{y}=M+C y-x^{2},
$$

appears for some classes of homoclinic tangencies of effective dimension $2[34,39,42,46,48]$. Note that the Mira map can be formally considered as a 3D Hénon map $\bar{x}=y, \bar{y}=z, \bar{z}=M+C z+B x-y^{2}$ with $B=0$. The 3D Hénon maps emerge in the study of homoclinic tangencies of effective dimension 3 (i.e. when three-dimensional volumes are not contracted by the map, see [34,46, 48,77-79]).

More general Hénon-like maps (with polynomial nonlinearities of order higher than two) appear near homoclinic tangencies of higher orders, as well as rescaled limits of multi-round maps near quadratic homoclinic tangencies $[32,33,42,44,45,47,81]$. A more general class of maps that emerge in a similar setting is given by compositions of Hénon-like maps. They were considered e.g. in [47,80,81]. An example of a composition of Hénon-like maps that emerge in the study of heteroclinic cycles of reversible systems is given by

$$
\left\{\begin{array}{l}
\bar{x}=M+c x-y^{2}, \\
\bar{y}=-\frac{M}{c}+\frac{1}{c} y+\frac{1}{c}\left(M+c x-y^{2}\right)^{2},
\end{array}\right.
$$

see $[82]$.

With the modern technique [48, 83, 84], the analysis of dynamics of the Henon-like maps can provide non-trivial results about the dynamics near homoclinic tangencies almost automatically. In particular, the birth of Lorenz-like attractors for many types of homoclinic tangencies was established in [49,77-79] based on the results of [1]. It was also proven in $[49,78,79]$ that infinitely many Lorenz-like attractors can coexist for generic maps from the Newhouse domain in the effective dimension 3 of more.

In Section 3, we give conditions which ensure the birth of small Lorenz-like attractors for the 3D Henon-like maps from a fixed point with the multipliers $(-1,-1,1)$ (at $B=1)$. The second iteration of the map near these small attractors is close (in the normal form coordinates) to a time-shift by the flow of the Shimizu-Morioka model. As $B$ decreases, the newly-born Lorenz-like attractor grows in size and, as we change parameters of the map, it may transform or get destroyed in variety of ways. We provide results of numerical experiments with several examples of the Henon-like maps, which show characteristic traits in the evolution and destruction of the Lorenz-like attractors. As our attractors are "genetically related" to the bifurcation $(-1,-1,1)$, the pictures of the orbits we obtain are often "flow-like" (see Figs. 4-6). In 
particular, the main stages of formation and destruction of the Lorenz-like attractor look similar to those in the Shimizu-Morioka model [21-23], with equilibria of the flow replaced by fixed or period-2 points of the map, and limit cycles of the flow replaced by closed invariant curves of the map. However, there are also clearly visible differences from the flow case. The main reason is that a closed invariant curve of the map typically behaves differently from a periodic orbit of the flow. While it can bifurcate similar to the way periodic orbits of a flow do (e.g. we observed cascades of doublings of invariant curves), it may also break down and transform to a "torus-chaos" attractor [85-91].

The Henon-like maps we consider here and the Poincare map for a celtic stone model considered in [17] are, so far, the only known examples of maps with Lorenz-like attractors. However, we think they should be universally present in weakly-dissipative three-dimensional maps. In particular, in all our examples here we observe the same scenario of the creation of Lorenz-like attractor: a stable fixed point becomes a saddle via the period-doubling bifurcation $\Rightarrow$ the emerged orbit of period 2 loses stability and an invariant pair of closed curves is born $\Rightarrow$ the curves grow in size and break down as the moment of homoclinic tangency for the now saddle fixed point is approached $\Rightarrow$ the chaotic set that contains homoclinic orbits to the fixed point transforms into a Lorenz-like attractor. This scenario was proposed in [92] as one of the most natural ways to chaos in maps in dimension three and higher (it is essential in this scenario that the map is not close to an area-contracting two-dimensional map: otherwise we can not have closed invariant curves, nor the volume-hyperbolic Lorenz-like attractor can form, see Section 2). Note that the ways of destruction of the Lorenz-like attractor we observe in different Henon-like maps may differ significantly: the attractor may become "bigger" and seemingly lose its volume-hyperbolic structure, or it may develop a lacuna where a stable closed invariant curve forms, which becomes a new attractor and, as parameters change, gets destroyed in one of several ways. We believe the gallery of attractor portraits which we produce here for main stages of the creation and destruction of Lorenz-like attractors in several examples of Henon-like maps should be a useful tool for detection of such attractors in various other models. In particular, the torus-chaos attractor of the shape close to that in Fig. 5(h) was found for a Poincare map of a model of celtic stone in [93], and it was then recognized in [17] as a clear signature of the presence of the Lorenz-like attractor in the same map.

\section{Lorenz-like attractors for diffeomorphisms}

Lorenz-like attractors are a subclass of pseudo-hyperbolic attractors. A theory of pseudohyperbolic attractors was built in $[18,19]$. The pseudo-hyperbolicity means that the following properties hold:

1) there are directions in which the dynamical system (a flow or a diffeomorphism) is strongly contracting ("strongly" means that any possible contraction in transverse directions is always strictly weaker); and

2) transverse to the contracting directions the system is volume-expanding (i.e. the volume is stretched exponentially).

The term volume-hyperbolicity may also be used in a similar context [94-96].

Definition 2.1. [18,19] A pseudohyperbolic attractor is a stable, chain-transitive, compact, invariant set which carries a uniformly pseudo-hyperbolic structure.

This definition is very wide. The class of systems with pseudo-hyperbolic attractors is $C^{r}$-open $(r \geq 2)$, and includes hyperbolic attractors, and Lorenz attractors as well: in the geometrical Lorenz model [2-8] (and in its modern variations $[97,98]$ ), in a neighbourhood of the attractor there is a contracting invariant foliation of codimension 2, and the flow expands area in the directions transverse to the foliation.

Let us describe the geometrical model of $[2,3]$ in more detail. In this model, we have a saddle equilibrium state $O$ with a one-dimensional unstable manifold $W^{u}(O)$ and a codimension-1 stable manifold $W^{s}(O)$. One assumes that there exists a cross-section $\Pi$ to $W^{s}$ such that all orbits of $\Pi \backslash W^{s}(O)$ return to $\Pi$, and both the unstable separatrices that constitute $W^{u}(O) \backslash O$ also intersect $\Pi$, see Fig. 2 . Thus, if we take the union of all forward orbits starting on $\Pi$ and the manifold $W^{u}(O)$, and take a small open neighbourhood $U$ of this union, then $U$ is an absorbing domain, i.e. any forward time shift of the closure of $U$ lies strictly 


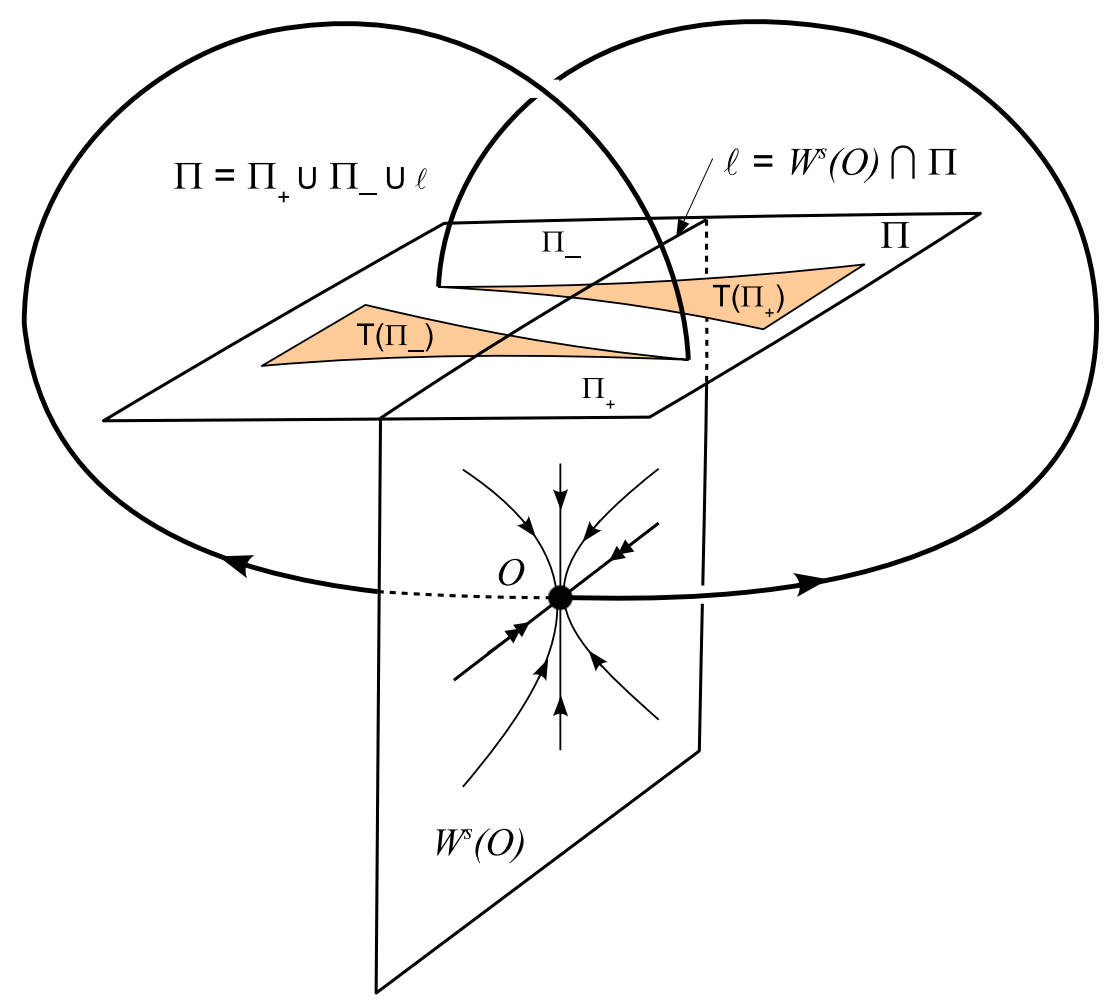

FiguRE 2. Poincare map for the geometrical Lorenz model.

inside $U$. The flow in $U$ defines the first-return map $T: \Pi \backslash W^{s}(O) \rightarrow \Pi$. Conditions given in [2,3] ensure the hyperbolicity of the map $T$ : this map has a contracting invariant foliation of codimension 1 , and it is expanding transverse to the foliation. This is not a true uniform hyperbolicity, as the first-return map $T$ has a singularity (discontinuity) at $\Pi \cap W^{s}(O)$. So, the theory of the Lorenz attractor is typically built in terms of the study of the maps with this kind of singular hyperbolic structure (and flow suspensions over such maps) [2-8,99-103]. For our purposes, it is important that the (singular) hyperbolicity of the first-return map is, in fact, equivalent to the uniform pseudohyperbolicity of the flow in $U$ (roughly speaking, one recovers a codimension 2 contracting invariant foliation of $U$ from the contracting invariant foliation on $\Pi$, and the expansivity of the first-return map in the direction transverse to the foliation on $\Pi$ means that the flow expands the area spanned by this direction and the phase velocity vector). By construction of the geometrical Lorenz model, $\Pi \backslash W^{s}(O)$ consists of two connected components, $\Pi_{+}$and $\Pi_{-}$, and the quotient of each of the maps $\left.T\right|_{\Pi_{+}}$and $\left.T\right|_{\Pi_{-}}$is injective. Using this fact, it was shown in [18] that if we denote as $A$ the set of all points that are attainable from $O$ by $\varepsilon$-orbits for arbitrarily small $\varepsilon$, then $A$ is chain-transitive. Moreover, this set is attainable by $\varepsilon$-orbits from every point in $U$, and it is stable under persistent perturbations (strictly stable in terms of [104]), i.e. given any $\delta>0$ there exist $\varepsilon>0$ such that no $\varepsilon$-orbit starting in $A$ can leave the $\delta$-neighbourhood of $A$. Thus, so defined set $A$ is the unique pseudohyperbolic attractor of the geometrical Lorenz model. We note that there exist cases when the geometric Lorenz model has no transitive attractor (this happens when the hyperbolic set inside a non-trivial lacuna hits the boundary of the transitive component, see [3]). Therefore, the notion of a chain-transitive attractor becomes useful in this situation. 
We note that there are many definitions of attractors, see [105-108]. We will stick to the notion of attractor based on the use of $\varepsilon$-orbits, this notion goes back to Conley, Ruelle, and Hurley [109-111]. We have just used this definition for the geometrical Lorenz model.

Definition 2.2. We call a compact invariant set an attractor if it is chain-transitive, i.e. every point of this set is attainable from any other point of it by $\varepsilon$-orbits that lie in the same set, for an arbitrarily small $\varepsilon$, and stable, in the sense that $\varepsilon$-orbits starting near this set always remain in some small neighbourhood of it.

The original idea of Conley was that these objects are most natural from the topological dynamics point of view. Ruelle had stressed that such defined attractors behave properly under the influence of a small bounded noise. These attractors were reinvented in [18] as they occur to be particularly fit for the description of volume-hyperbolic dynamics. The stability property of these attractors can be formulated in several equivalent ways.

For example, in the spirit of $[104,112]$ we may define the $C^{0}$-prolongation of a point in the phase space as the set of all points attainable from it by $\varepsilon$-orbits for an arbitrarily small $\varepsilon$ (the $C^{0}$-prolongation of a set is the union of the $C^{0}$-prolongations of its points; an invariant set always belongs to its own prolongation, along with its unstable set). It is easy to see that

the $C^{0}$-prolongation of any point/set is always a stable invariant closed set $[18,104,109-111]$. Hence, a chain-transitive attractor equals to the $C^{0}$-prolongation of any of its point.

This provides us with the working strategy of finding the attractors:

take a prolongation of a suitable point and try to see if it is chain-transitive.

Another characterisation of stable sets is that the set is stable if and only if it is an intersection of at most countable set of nested absorbing domains [18,109-111]. Thus, given any system, for any attractor there exists at least one absorbing domain, and every absorbing domain contains at least one attractor as we defined it. In general, there is no one-to-one correspondence between chain-transitive attractors and their absorbing domains. However, for pseudohyperbolic systems there exist effectively verifiable criteria of finiteness of the number of attractors, or even of uniqueness of the attractor [19].

In [19], a geometrical Lorenz model of [2,3] subject to a small nonautonomous time-periodic forcing was considered. It was shown that the properties of pseudohyperbolicity and chain-transitivity of the non-perturbed Lorenz attractor are inherited by the periodically perturbed one. Therefore, the Poincare map (the map for the period of the forcing) has here a pseudohyperbolic attractor $A$, which we will call Lorenz-like. It is a genuine strange attractor, as it does not contain stable periodic points and this holds true for every $C^{r}$-close map as well (because the pseudohyperbolicity is a $C^{r}$-open property, and the second of the pseudohyperbolicity conditions, i.e. the volume-expansion property, implies the positivity of the maximal Lyapunov exponent of every orbit; this was also shown in [113]). The saddle equilibrium in the Lorenz attractor of the non-perturbed flow corresponds to a saddle fixed point of the Poincare map of the periodically forced system. The attractor $A$ is exactly the set of all points which can be reached by forward $\varepsilon$-orbits starting at the fixed point for every $\varepsilon>0$, and this set is always chain-transitive (when the forcing term is small), see [19].

Absolutely the same conclusions can be derived without the assumption that the map under consideration is the Poincare map of a time-periodic flow close to an autonomous one (i.e. we do not need to assume that our map is a small perturbation of the constant-time shift by an autonomous flow of the geometrical Lorenz model). Namely, one can give a definition of the Lorenz-like attractor for a map $f$ as follows.

Definition 2.3. Let $f$ have a periodic point $O$ with a one-dimensional unstable manifold. Consider a smooth flow suspension $F_{t}$ over $f$. The point $O$ corresponds to a periodic orbit $L$ of $F$, with a twodimensional unstable manifold $W^{u}(L)$.

(i) Suppose the flow $F$ has a cross-section $\hat{\Pi}$ to the stable manifold $W^{s}(L)$ such that the forward orbit of each point in $W^{u}(L)$ intersects $\hat{\Pi}$, and each forward orbit that starts on $\hat{\Pi} \backslash W^{s}(L)$ returns on $\hat{\Pi}$. 
Then the set $D$ which is a small neighbourhood of the union of $W^{s}(L)$ with the union of all forward orbits of $F$ starting on $\hat{\Pi}$ is an absorbing domain, i.e. $F_{t}(c l D) \subset$ int $D$ for all $t>0$.

(ii) Let $F_{t}$ be uniformly pseudohyperbolic in $D$.

(iii) Let $\hat{\Pi} \backslash W^{s}(L)$ consist of two connected components, $\hat{\Pi}_{+}$and $\hat{\Pi}_{-}$.

The flow $F_{t}$ defines the first-return map $T: \hat{\Pi}_{+} \cup \hat{\Pi}_{-} \rightarrow \hat{\Pi}$. The pseudohyperbolicity of the flow $F_{t}$ implies the pseudohyperbolicity of the first-return map $T$. In particular, it implies the existence of an absolutely continuous strong-stable invariant foliation tangent to the strongly contracting directions, and it also implies that the quotient of the map $T$ by this foliation is area-expanding.

(iv) Assume that the restrictions of the quotient map on $\hat{\Pi}_{+}$and on $\hat{\Pi}_{-}$are injective.

Under these conditions the chain-transitive attractor $\tilde{A} \subset D$ is unique and coincides with the set of points attainable by forward $\varepsilon$-trajectories from $L$ for every $\varepsilon>0$ [19]. The restriction of $\tilde{A}$ to the base of the flow suspension is the chain-transitive pseudo-hyperbolic attractor $A$ of the map $f$. We will call the attractor $A$ that emerges in this way a pseudohyperbolic Lorenz-like attractor. Under the above described conditions, the attractor $A$ is the set of all points attainable by the forward $\varepsilon$-trajectories from the periodic point $O$ for every $\varepsilon>0$.

As one can easily see, the conditions of this definition are satisfied e.g. by the constant-time shift map by the flow of the geometrical Lorenz model of $[2,3]$ (to define a suspension flow for this map one adds a time coordinate $\theta \in S^{1}$ with a trivial dynamics in $\theta$, i.e. $\dot{\theta}=1$, then one defines $D=U \times S^{1}$ and $\hat{\Pi}=\Pi \times S^{1}$, where $U$ and $\Pi$ are the absorbing domain and the cross-section for the geometrical Lorenz model, as described above). It is also easy to see that these conditions hold for all $C^{r}$-small perturbations of the map.

Except for the positivity of the maximal Lyapunov exponent for every orbit, it is hard to say anything more about the dynamics of a such defined Lorenz-like attractor of a general map. However, the case of a small perturbation of the time- 1 shift of the Lorenz flow can be studied in much more detail. Thus, it was shown in [19] that given any autonomous flow with the geometrical Lorenz attractor an arbitrary small non-autonomous periodic perturbation can be constructed such that the chain-transitive pseudohyperbolic Lorenz-like attractor $A$ for the Poincare map of the perturbed system contains a Newhouse wild-hyperbolic subset along with its unstable manifold (and this property is $C^{r}$-open).

We recall that according to [10-12], a wild hyperbolic set is a uniformly-hyperbolic basic set whose stable and unstable manifolds $W^{u}$ and $W^{s}$ have a persistent tangency, i.e. the stable and unstable manifolds of a wild hyperbolic set have a nontransverse intersection, both for the system itself and for any $C^{r}$-close system, $r \geq 2$. As Newhouse proved, systems with wild hyperbolic sets comprise non-empty $C^{r}$-open sets in the space of dynamical systems (we call them Newhouse regions). Choose a wild-hyperbolic set $\Lambda$, and denote as $A \supset W^{u}(\Lambda)$ the set of all points which, for every $\varepsilon>0$, can be reached by $\varepsilon$-orbits starting at $\Lambda$. The uniformly-hyperbolic compact invariant set $\Lambda$ varies continuously when we add $C^{r}$ small perturbations to the system, but the prolongation $A$ does not need to be a continuous function of the system. However, in some cases $A$ remains chain-transitive for the system itself and for every $C^{r}$-close system. In this case we call $A$ a wild-hyperbolic attractor. Namely, we adhere to the following definition.

Definition 2.4. A wild-hyperbolic attractor is a $C^{r}$-robustly chain-transitive prolongation of a wild hyperbolic set.

The notion of wild pseudohyperbolic attractor was introduced in [18]. A first example of a "spiral" wild-hyperbolic attractor for flows in the space of dimension 4 or higher was also constructed there. A different example was constructed in [114]. The above described result from [19] of the wildness of the attractors of certain non-autonomous periodic perturbations of the Lorenz flow shows that the dynamics of Lorenz-like attractors of smooth maps is much richer than that of the Lorenz attractors for autonomous flows. Namely, it follows from $[18,19,32-34,42,44-48]$ that

the wild Lorenz-like attractors may contain orbits of homoclinic tangency of an arbitrarily high order along with arbitrarily-degenerate periodic orbits. 
Note that the problem of proving that a given Lorenz-like attractor is wild is not trivial. It is not enough to show the existence of a wild hyperbolic set somewhere in the absorbing domain $D$. In order to claim that the wild set belongs to the attractor $A$, one needs to show that the wild set is attainable from the "basic" saddle periodic point $O$ by $\varepsilon$-orbits for arbitrarily small $\varepsilon$. The simplest way of doing that is to prove that the point $O$ itself belongs to the wild set. This is done by showing that $O$ has a homoclinic tangency. Then the multidimensional version $[35,36]$ of the Newhouse theorem [12] allows to show that $O$ becomes an element of a wild-hyperbolic set after a small perturbation of the map [19].

In fact, more results about the dynamics of the Lorenz-like attractor follow from the existence of a homoclinic tangency. By $[34,41,46,48]$, as the orbit of homoclinic tangency to $O$ is volume-hyperbolic, the tangency can split in such a way that the map acquires a periodic orbit with a two-dimensional unstable manifold. With an extra effort one can show [19] that such periodic orbits can belong to the attractor, i.e. they are attainable from $O$ by $\varepsilon$-orbits. In fact, using the construction of heterodimensional cycles $[95,96,115-117,120]$, one can prove that in the $C^{r}$-open set of maps with a wild Lorenz-like attractor there is an open and dense subset such that for every map that belongs to this set the Lorenz-like attractor contains saddle periodic orbits with two-dimensional unstable manifolds. Recall that the Lorenz-like attractor always contains saddles with one-dimensional unstable manifolds, the point $O$ for example. Thus, a $C^{r}$-generic wild Lorenz-like attractor is heterodimensional in the sense of the following

Definition 2.5. Lorenz-like attractors that contain simultaneously saddle periodic orbits with different dimensions of unstable manifolds are called heterodimensional Lorenz-like attractors.

Thus, a small time-periodic perturbation of a Lorenz attractor of an autonomous flow creates a pseudohyperbolic Lorenz-like attractor for the Poincare map. Moreover, for an open class of perturbations this attractor will be heterodimensional and wild, i.e. it will exhibit dynamical features which have no analogues in the autonomous Lorenz attractor. Another dynamical property of periodic perturbations of Lorenz attractors is that the resulting Lorenz-like attractors may contain saddle invariant tori. Indeed, the Lorenz attractor for an autonomous flow contains non-trivial hyperbolic sets where saddle periodic orbits are dense [3]. The hyperbolic set for a flow is a partially-hyperbolic set for the constant-time shift map by the flow (denote this set as $\Omega$ ), whereas the saddle periodic orbits become saddle closed invariant curves for this map. The Poincare map for a $C^{r}$-small $\tau$-periodic perturbation of the flow is a $C^{r}$-small perturbation to the time- $\tau$ shift map, such perturbations do not destroy the partially-hyperbolic set $\Omega$, and the saddle closed invariant curves also preserve and remain dense in $\Omega$. Partially-hyperbolic sets of this structure were first studied by Shilnikov in [121] (see also [122-125]), so we will here call them Shilnikov sets. One may prove that for an open set of small periodic perturbations of a flow with a Lorenz attractor some Shilnikov set $\Omega$ will be attainable by $\varepsilon$-orbits from $O$, i.e. the Lorenz-like attractor for the Poincare map will contain this set (cf. [146]).

In general, we introduce the following

Definition 2.6. Consider an arbitrary diffeomorphism with a Lorenz-like attractor. Let this attractor $C^{r}$-robustly contain a partially-hyperbolic Shilnikov set $\Omega$. Consider the flow suspension over the diffeomorphism (see Definition 2.3), and let the intersections of the saddle invariant tori from $\Omega$ with the cross-section $\hat{\Pi}$ be not contractible in $\hat{\Pi}$ to a point. Moreover, assume that these intersections are, each, a finite union of closed curves homotopic to $W^{s}(L) \cap \hat{\Pi}$ in $\hat{\Pi}$ (i.e. they are similar to the saddle invariant tori of a small time-periodic perturbation of a Lorenz attractor). In this case we will call this attractor a true Lorenz-like attractor.

Notice that the true Lorenz-like attractor is also heterodimensional, as it contains, along with the one-dimensional unstable manifold of the saddle point $O$, the two-dimensional unstable manifold of the Shilnikov set.

Let us summarise the above described theory in the following terminology table. 


\begin{tabular}{|c|c|}
\hline Attractor & See Definition 2.2 \\
\hline Pseudohyperbolic attractor & See Definition 2.1 \\
\hline Lorenz attractor & $\begin{array}{l}\text { The chain-recurrent pseudohyperbolic attractor of the flow } \\
\text { of the geometrical Lorenz model of }[2,3,18]\end{array}$ \\
\hline Lorenz-like attractor & $\begin{array}{l}\text { The chain-recurrent pseudohyperbolic attractor of a diffeo- } \\
\text { morphism (e.g. of a Poincare map of a flow), which satisfies } \\
\text { the conditions of Definition } 2.3\end{array}$ \\
\hline Wild Lorenz-like attractor & $\begin{array}{l}\text { A Lorenz-like attractor that contains a wild hyperbolic set } \\
\text { (see Definition 2.4) }\end{array}$ \\
\hline Heterodimensional Lorenz-like attractor & See Definition 2.5 \\
\hline True Lorenz-like attractor & $\begin{array}{l}\text { A version of heterodimensional attractor given by } \\
\text { Definition } 2.6\end{array}$ \\
\hline
\end{tabular}

As we see, the Lorenz-like attractors provide a very rich example of multidimensional strange attractors. Importantly, they can be created by local bifurcations of periodic orbits; in particular, when the periodic orbit has three or more Floquet multipliers on the unit circle, the normal form can be a time-periodic perturbation of a system with a Lorenz attractor [23]. Thus, Lorenz-like attractors can easily appear in concrete models when there is enough parameters to permit the degenerate periodic orbits of this type.

In particular, let a diffeomorphism $f$ have a fixed point $O$ with the multipliers $(-1,-1,+1)$. According to [23] (see also [126]), in a generic three-parameter unfolding of this bifurcation there is a region of parameter values for which the second iteration of the map $f$ near the bifurcating point is $o(\tau)$-close to the time- $\tau$ map of the Shimizu-Morioka system

$$
\dot{X}=Y, \quad \dot{Y}=X(\mu-Z)-\lambda Y, \quad \dot{Z}=-\alpha Z+X^{2},
$$

for $\tau$ which can be taken as small as we want, $\mu= \pm 1$, and $\alpha, \lambda$ taking arbitrary positive values (see examples in the next Section).

It was shown numerically in [21-23] that system (2.1) at $\mu=1$ possesses a Lorenz attractor for some domain of positive parameter $(\alpha, \lambda)$. Therefore, for a certain open region of parameter values the original map $f$ has an attractor which is a $\tau$-periodic perturbation of a Lorenz attractor. We give a detailed computation of the normal form near $(-1,-1,1)$ bifurcation for the Henon-like maps in the next Section. We provide a formula for computing the normal form coefficient $\mu$ in terms of the Taylor expansion of the original map at the fixed point at the moment of bifurcation, thus providing (Lemma 3.1) a criterion of the birth of a Lorenz-like attractor in the Henon-like maps.

We stress that the crucial fact that the Shimizu-Morioka system has a Lorenz attractor is reliably verified numerically and hardly causes any doubt, however a fully rigorous proof still does not exist. In order to obtain a free of computer assistance proof of the existence of the Lorenz attractor in the Shimizu-Morioka model (and, hence, the pseudohyperbolic Lorenz-like attractor in the Henon-like maps satisfying criterion (3.3)), one can use one of the criteria of the birth of the Lorenz attractor from a pair of homoclinic loops which were given in [127-129]. In particular, the domain of existence of the Lorenz attractor adjoins to a point in the parameter plane where the system has a pair of symmetric homoclinic loops to a saddle with zero saddle value, provided the so-called separatrix value is non-zero and less than 2 in the absolute value (see definitions in [84]). The existence of such point in the plane of parameters $(\alpha, \lambda)$ for the Shimizu-Morioka system with $\mu=1$ was proven analytically in [130]. While it was checked numerically in [21-23] that the separatrix value lies in $(0,1)$ at this point (hence the conditions of [127] for the birth of the Lorenz attractor are satisfied), the analytical estimate for the separatrix value was not obtained in [130]. Thus, this estimate is the only thing remaining for a complete removal of the numerical evidence here. To precisely describe the situation, we make the following 
Claim 2.1. The Shimizu-Morioka system (2.1) at $\mu=1$ has, at a positive value of $(\alpha, \lambda)$, a pair of symmetric homoclinic loops to the saddle at $X=Y=Z=0$ with zero saddle value. The separatrix value is positive and less than 1 . As parameters change the homoclinic loops split in such a way that a Lorenz attractor is born.

As we just said the bounds on the separatrix value were not obtained analytically. So this part of the claim should, perhaps, be considered as a conjecture, supported by a reliable numerics.

\section{Normal forms and numerics}

In this Section we give conditions for the birth of Lorenz-like attractors in the three-dimensional Henonlike maps, and investigate numerically the ways such attractor evolves as parameters change. We consider the maps $(x, y, z) \mapsto(\bar{x}, \bar{y}, \bar{z})$ such that

$$
\bar{x}=y, \quad \bar{y}=z, \quad \bar{z}=B x+f(y, z),
$$

where $f$ is a smooth function. The fixed points of the map are given by

$$
x=y=z, \quad x(1-B)=f(x, x) .
$$

The characteristic equation at the fixed point $x=y=z=x_{0}$ is

$$
\lambda^{3}-A \lambda^{2}-C \lambda-B=0,
$$

where $A=f_{z}^{\prime}\left(x_{0}, x_{0}\right), C=f_{y}^{\prime}\left(x_{0}, x_{0}\right)$. At $(A=-1, C=1, B=1)$, the fixed point has multipliers $(+1,-1,-1)$. Let us now derive a normal form that describes bifurcations of this point.

Consider a smooth three-parameter family of maps (3.1) which, at zero parameter values, has a fixed point with multipliers $(1,-1,-1)$, and assume the fixed point exists for a certain region of parameter values adjoining to zero. Move the origin to the fixed point. The map will take the form

$$
\bar{x}=y, \quad \bar{y}=z, \quad \bar{z}=\left(1-\varepsilon_{1}\right) x+\left(1-\varepsilon_{2}\right) y-\left(1+\varepsilon_{3}\right) z+a y^{2}+b y z+c z^{2}+\ldots,
$$

where $\varepsilon_{1,2,3}$ are small,

$$
a=\frac{1}{2} f_{y y}^{\prime \prime}\left(x_{0}, x_{0}\right), \quad b=f_{y z}^{\prime \prime}\left(x_{0}, x_{0}\right), \quad c=\frac{1}{2} f_{z z}^{\prime \prime}\left(x_{0}, x_{0}\right),
$$

and the dots stand for cubic and higher order terms. Let the coefficients $a, b$ and $c$ depend on $\varepsilon_{1,2,3}$ smoothly.

Lemma 3.1. Let the following condition hold at $\varepsilon=0$ :

$$
(c-a)(a-b+c)>0 .
$$

Then Claim 2.1 implies that map (3.2) has a pseudohyperbolic Lorenz-like attractor for all $\varepsilon$ from an open, adjoining to $\varepsilon=0$, subregion $\mathcal{D}$ of $\left\{\varepsilon_{1}>0, \varepsilon_{1}+\varepsilon_{3}>0,\left|\varepsilon_{2}-\varepsilon_{1}-\varepsilon_{3}\right| \leq L\left(\varepsilon_{1}^{2}+\varepsilon_{3}^{2}\right)\right\}$, for some $L>0$.

Proof. Let $\nu$ be the multiplier of the zero fixed point of map (3.2) which is close to 1 :

$$
\nu=1-\frac{\varepsilon_{1}+\varepsilon_{2}+\varepsilon_{3}}{4}+O\left(\|\varepsilon\|^{2}\right) .
$$

Let us make the following linear coordinate transformation:

$$
u_{1}=-\nu x+y, \quad u_{2}=\nu x+(\nu-1) y-z, \quad u_{3}=\nu\left(1-\varepsilon_{1}\right) x+\left(1-\varepsilon_{1}+\nu\left(1-\varepsilon_{2}\right)\right) y+\nu^{2} z .
$$


Map (3.2) takes the following form

$$
\begin{aligned}
& \bar{u}_{1}=-u_{1}-u_{2}, \\
& \bar{u}_{2}=-\delta_{1} u_{1}+\left(-1+\delta_{2}\right) u_{2}-\frac{1}{16}\left(a\left(2 u_{1}+u_{2}+u_{3}\right)^{2}-b\left(2 u_{1}+u_{2}+u_{3}\right)\left(2 u_{1}+3 u_{2}-u_{3}\right)+\right. \\
& \left.+c\left(2 u_{1}+3 u_{2}-u_{3}\right)^{2}\right)+O\left(\|u\|^{2}\|\varepsilon\|+\|u\|^{3}\right), \\
& \bar{u}_{3}=\left(1-\delta_{3}\right) u_{3}+\frac{1}{16}\left(a\left(2 u_{1}+u_{2}+u_{3}\right)^{2}-b\left(2 u_{1}+u_{2}+u_{3}\right)\left(2 u_{1}+3 u_{2}-u_{3}\right)+\right. \\
& \left.+c\left(2 u_{1}+3 u_{2}-u_{3}\right)^{2}\right)+O\left(\|u\|^{2}\|\varepsilon\|+\|u\|^{3}\right),
\end{aligned}
$$

where

$$
\begin{aligned}
& \delta_{1}=\nu+\varepsilon_{3}-\frac{1-\varepsilon_{1}}{\nu}=\frac{\varepsilon_{1}-\varepsilon_{2}+\varepsilon_{3}}{2}+O\left(\|\varepsilon\|^{2}\right), \\
& \delta_{2}=1-\nu-\varepsilon_{3}=\frac{\varepsilon_{1}+\varepsilon_{2}-3 \varepsilon_{3}}{4}+O\left(\|\varepsilon\|^{2}\right), \\
& \delta_{3}=1-\nu=\frac{\varepsilon_{1}+\varepsilon_{2}+\varepsilon_{3}}{4}+O\left(\|\varepsilon\|^{2}\right) .
\end{aligned}
$$

Note that the linear part of (3.4) is in the Jordan form at $\varepsilon=0$.

The terms $u_{1}^{2}, u_{2}^{2}, u_{1} u_{2}$ in the second equation, and the terms $u_{1} u_{3}, u_{2} u_{3}$ in the third equation are non-resonant, so they can be killed by a close to identity quadratic change of coordinates. After that the system takes the form

$$
\begin{aligned}
& \bar{u}_{1}=-u_{1}-u_{2}, \\
& \bar{u}_{2}=-\delta_{1} u_{1}+\left(-1+\delta_{2}\right) u_{2}-\frac{u_{3}}{8}\left(2 u_{1}(a-c)+u_{2}(a-b-3 c)\right)+O\left(\|u\|^{2}\|\varepsilon\|+\|u\|^{3}\right), \\
& \bar{u}_{3}=\left(1-\delta_{3}\right) u_{3}+\frac{1}{16}\left(u_{3}^{2}(a+b+c)+a\left(2 u_{1}+u_{2}\right)^{2}-b\left(2 u_{1}+u_{2}\right)\left(2 u_{1}+3 u_{2}\right)+\right. \\
& \left.\quad+c\left(2 u_{1}+3 u_{2}\right)^{2}\right)+O\left(\|u\|^{2}\|\varepsilon\|+\|u\|^{3}\right),
\end{aligned}
$$

One checks that the composition of map (3.5) with the involution

$$
S:\left(u_{1}, u_{2}, u_{3}\right) \leftrightarrow\left(-u_{1},-u_{2}, u_{3}\right)
$$

is a map whose linear part at the origin has the Jordan form $\left(\begin{array}{lll}1 & 1 & 0 \\ 0 & 1 & 0 \\ 0 & 0 & 1\end{array}\right)$ at $\varepsilon=0$ and coincides, up to terms of order $O\left(\|u\|^{2}\|\varepsilon\|+\|u\|^{3}\right)$, with the time-1 shift by the flow

$$
\begin{aligned}
& \dot{u}_{1}=\hat{\rho}_{1} u_{1}+\left(1+\hat{\rho}_{2}\right) u_{2}-\frac{1}{8} u_{1} u_{3}(a-c)-\frac{1}{48} u_{2} u_{3}(a-3 b-7 c), \\
& \dot{u}_{2}=\rho_{1} u_{1}-\rho_{2} u_{2}+\frac{1}{4} u_{1} u_{3}(a-c)+\frac{1}{8} u_{2} u_{3}(b+2 c), \\
& \dot{u}_{3}=-\rho_{3} u_{3}+\frac{1}{4} u_{1}^{2}(a-b+c)+\frac{1}{2} u_{1} u_{2}(b+c)-\frac{1}{48} u_{2}^{2}(a-b-11 c)+\frac{1}{16} u_{3}^{2}(a+b+c) .
\end{aligned}
$$

where

$$
\begin{gathered}
\rho_{3}=-\ln \left(1-\delta_{3}\right)=\frac{\varepsilon_{1}+\varepsilon_{2}+\varepsilon_{3}}{4}+O\left(\|\varepsilon\|^{2}\right), \\
\left(\begin{array}{cc}
\hat{\rho}_{1} & 1+\hat{\rho}_{2} \\
\rho_{1} & -\rho_{2}
\end{array}\right)=\ln \left(\begin{array}{cc}
1 & 1 \\
\delta_{1} & 1-\delta_{2}
\end{array}\right) .
\end{gathered}
$$

The latter implies

$$
\begin{aligned}
& \hat{\rho}_{1}=-\frac{\varepsilon_{1}-\varepsilon_{2}+\varepsilon_{3}}{4}+O\left(\|\varepsilon\|^{2}\right), \quad \hat{\rho}_{2}=\frac{7 \varepsilon_{1}-\varepsilon_{2}-5 \varepsilon_{3}}{24}+O\left(\|\varepsilon\|^{2}\right), \\
& \rho_{1}=\frac{\varepsilon_{1}-\varepsilon_{2}+\varepsilon_{3}}{2}+O\left(\|\varepsilon\|^{2}\right), \quad \rho_{2}=\frac{\varepsilon_{1}-\varepsilon_{3}}{2}+O\left(\|\varepsilon\|^{2}\right) .
\end{aligned}
$$

Since system (3.7) is invariant with respect to involution (3.6), it follows that the square of map (3.5) coincides with the time-2 shift by the flow of system (3.7) up to terms of order $O\left(\|u\|^{2}\|\varepsilon\|+\|u\|^{3}\right)$. 
Let us now scale the coordinates $\left(u_{1}, u_{2}, u_{3}\right)$, the time $t$, and the parameters as follows:

$$
t=\tau / s, \quad u_{1}=4 s^{3 / 2} X, \quad u_{2}=4 s^{5 / 2} Y, \quad u_{3}=-4 s^{2} Z, \quad \rho_{2}=s \lambda, \quad \rho_{3}=s \alpha,
$$

where the small scaling factor $s$ is

$$
s=\sqrt{\rho_{1}} .
$$

We are interested in values of $(\alpha, \lambda)$ in a bounded domain of the positive quadrant. This implies that we consider our system in the region of parameters $\varepsilon$ where the values $\rho_{1,2,3}$ are positive and $\rho_{1}=$ $O\left(\left\|\rho_{2}\right\|^{2},\left\|\rho_{3}\right\|^{2}\right)$, i.e. $\varepsilon_{2}=\varepsilon_{1}+\varepsilon_{3}+O\left(\varepsilon_{1}^{2}+\varepsilon_{3}^{2}\right)$, and $\varepsilon_{1}>0, \varepsilon_{1}+\varepsilon_{3}>0$. Since $s$ becomes arbitrarily small as we approach the bifurcation point $\varepsilon=0$ in the initial parameter space, it follows that the rescaled coordinates $(X, Y, Z)$ and the new parameters $\lambda$ and $\mu$ can take arbitrary finite values.

After the scaling, system (3.7) takes the form

$$
\begin{aligned}
& \dot{X}=Y+O(s), \\
& \dot{Y}=X(1-(a-c) Z)-\lambda Y+O(s), \\
& \dot{Z}=-\mu Z-(a-b+c) X^{2}+O(s) .
\end{aligned}
$$

where the dot denotes $d / d \tau$. By (3.3), we may introduce new variables $Z_{\text {new }}=(a-c) Z, X_{\text {new }}=$ $\sqrt{(c-a)(a-b+c)} X, Y_{\text {new }}=\sqrt{(c-a)(a-b+c)} Y$, and the system takes the form

$$
\begin{aligned}
& \dot{X}=Y+O(s), \\
& \dot{Y}=X(1-Z)-\lambda Y+O(s), \\
& \dot{Z}=-\alpha Z+X^{2}+O(s),
\end{aligned}
$$

Since the difference between the second iterate of map (3.5) and the time-2 shift by the flow of system (3.7) is $O\left(\|u\|^{2}\|\varepsilon\|+\|u\|^{3}\right)=O\left(s^{4}\right)$, we find that in the rescaled coordinates the second iterate of (3.5) is $O\left(s^{3 / 2}\right)$-close to the time- $2 s$ shift by the flow of (3.10). It follows that when we write map (3.5) in the coordinates $(X, Y, Z)$, its $N$-th iteration is $O(\sqrt{s})$-close to the time- 1 shift by the flow of $(3.10)$, where $N / 2$ equals to the integral part of $1 /(2 s)$. Recall that the pseudohyperbolic Lorenz-like attractors preserve at small perturbations [19]. Therefore, we will prove that map (3.5) indeed has such attractor at all small $s$, if we prove that system (3.10) has, at all small $s$, a Lorenz attractor for a certain, independent of $s$, region of $(\alpha, \lambda)$-values (see [1] for more discussions).

Now note that at $s=0$ system (3.10) is the Shimizu-Morioka system. By Claim 2.1, we may assume that it has a Lorenz attractor in an open domain of the $(\mu, \lambda)$-plane. The existence of a Lorenz attractor is a robust property [3], i.e. there exists a region of the $(\mu, \lambda)$-values such that every close enough system, e.g. system (3.10) at all small $s$, has to have a Lorenz attractor at $(\mu, \lambda)$ from this region.

Note that lemma holds true, by the robustness of pseudohyperbolicity, for any family close to (3.2) in the space of continuous families of $C^{r}$-smooth maps $(r \geq 2)$. Moreover, one can show that arbitrarily close (in $\left.C^{r}, r=2, \ldots, \infty\right)$ to family (3.2) there exists an open set of families such that the Lorenz-like attractor is true, heterodimensional and wild for an open set of values of $\varepsilon \in \mathcal{D}$. This is done, essentially, by proving that a quadratic homoclinic tangency to the fixed point at zero can be created by a small perturbation (cf. [19]), we will not pursue this topic here.

The first example to lemma 3.1 is given by the map

$$
\bar{x}=y, \quad \bar{y}=z, \quad \bar{z}=M+B x+C y-z^{2}
$$

(see $[1,77])$. At $(M=-1 / 4, B=1, C=1)$ the map has a fixed point $x=y=z=\frac{1}{2}$. After shifting the coordinate origin to this point we will have the map in the form

$$
\bar{x}=y, \quad \bar{y}=z, \quad \bar{z}=x+y-z-z^{2},
$$


i.e. the fixed point has multipliers $(1,-1,-1)$, and the coefficients $a, b, c$ are

$$
a=0, \quad b=0, \quad c=-1 .
$$

As we see, condition (3.3) of the lemma holds.

The second example is given by the map

$$
\bar{x}=y, \quad \bar{y}=z+c y^{2}, \quad \bar{z}=M_{0}+B x+M_{1} y+A z+d y^{3}+b y z .
$$

Introduce $z_{\text {new }}=z+c y^{2}$. Then, map (3.12) takes the standard Henon form

$$
\bar{x}=y, \quad \bar{y}=z, \quad \bar{z}=M_{0}+B x+M_{1} y+A z-A c y^{2}+c z^{2}+\beta y z+(d-b c) y^{3} .
$$

Let $x=y=z=x_{0}$ be a fixed point of (3.12), i.e.

$$
M_{0}=x_{0}\left(1-B-M_{1}-A\right)-(d-b c) x_{0}^{3}-(1+b c-A c) x_{0}^{2} .
$$

By shifting the coordinate origin to this point, we write the map in form (3.2):

$\left.\bar{x}=y, \quad \bar{y}=z, \quad \bar{z}=B x+\left(M_{1}+(b-2 A c) x_{0}+3(d-b c) x_{0}^{2}\right) y+(A+b+2 c) x_{0}\right) z+a y^{2}+b y z+c z^{2}+\ldots$ where

$$
a=3(d-b c) x_{0}-A c .
$$

The fixed point has the multipliers $(1,-1,-1)$ at

$$
B=1, \quad A+x_{0}(b+2 c)=-1, \quad M_{1}=1+(2 A c-b) x_{0}-3(d-b c) x_{0}^{2} .
$$

Condition (3.3) reads as

$$
x_{0}\left(3 d-2 b c+2 c^{2}\right)\left[\left(3 d-2 b c+2 c^{2}\right) x_{0}-b+2 c\right]<0 .
$$

For every given $b, c$ and $d$ such that

$$
d \neq \frac{2}{3} c(c-b), \quad b \neq 2 c
$$

one can always find $x_{0}$ for which (3.14) is fulfilled. Therefore, by Lemma 3.1, for every fixed $b, c, d$ which satisfy (3.15), there is an open region in the space of parameters $\left(M_{0}, M_{1}, B, A\right)$ which corresponds to the existence of the Lorenz-like attractor.

Below we present results of a numerical study of Lorenz-like attractors of the maps from the examples above. We consider, first, map (3.11) with $B=0.7$ (i.e. at not a very small distance from the bifuraction point at $B=1$ ), and recall some numerical results from $[1,92]$. In Figure 3 we recall the "map of Lyapunov exponents" from [1]). This is a partition of the domain $D_{1}:\left\{-0.15<M_{1}<0.1 ; 0.7<M_{2}<1\right\}$ into areas corresponding to different types of the spectrum of numerically computed Lyapunov exponents $\Lambda_{1,2,3}$. Let $\Lambda_{1} \geq \Lambda_{2} \geq \Lambda_{3}$, then

1. green color (No. 1) corresponds to $\Lambda_{1}<0$, i.e. periodic attractor;

2. light blue color (No. 2) corresponds to $\Lambda_{1}=0>\Lambda_{2}>\Lambda_{3}$, e.g. a stable closed invariant curve;

3. yellow color (No. 3) corresponds to $\Lambda_{1}>0>\Lambda_{2}>\Lambda_{3}$;

4. red color (No. 4) corresponds to $\Lambda_{1}>0=\Lambda_{2}>\Lambda_{3}$;

5. (deep) blue color (No. 5) corresponds to $\Lambda_{1}>\Lambda_{2}>0>\Lambda_{3}$ 


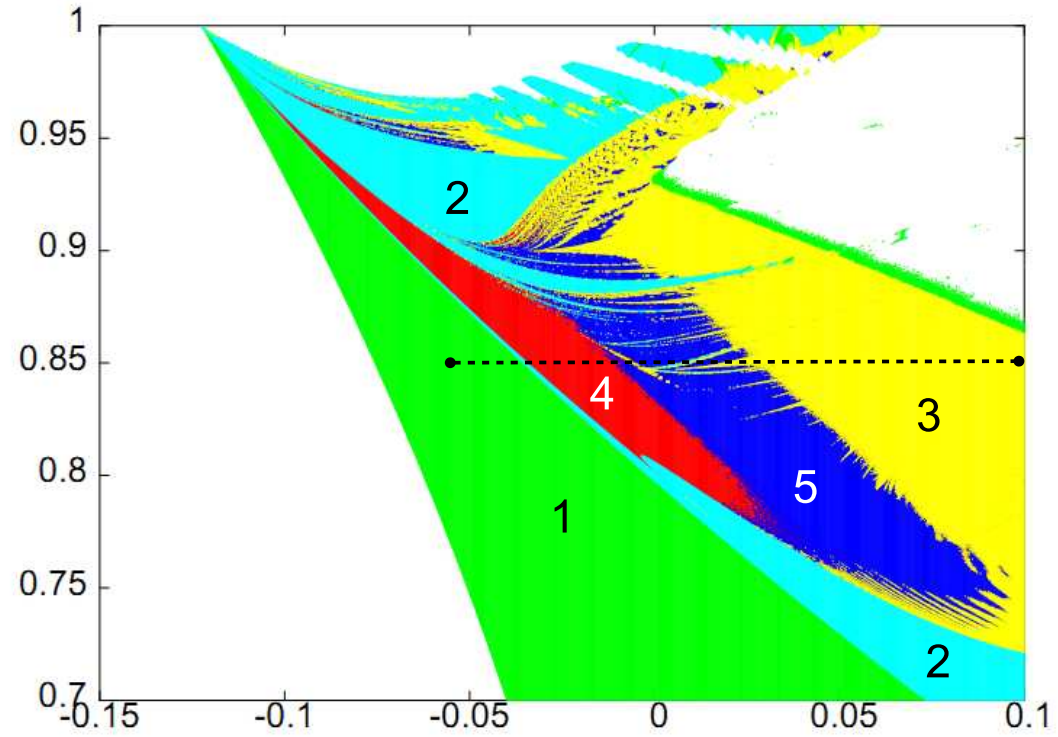

FiguRE 3. The map of Lyapunov exponents (from [1]) in the domain $D_{1}:\left\{-0.15<M_{1}<0.1 ; 0.7<\right.$ $\left.M_{2}<1\right\}$ for map (3.11) with $B=0.7$. See the color codes and numbering in the text.

(the white areas correspond to those values of the parameters $M_{1}$ and $M_{2}$ for which iterations of a chosen initial point escape to infinity). One interprets the last 3 cases in this list as corresponding to some type of a strange attractor. The sign "=" (the red color) means that the numerically obtained $\Lambda_{2}$ differs from 0 not more than the error bound of the numerical method $\left(10^{-6}\right.$ in [1]).

This Lyapunov map guides us in tracing the evolution, as the parameters change, of attracting sets of the map from a simple attractor (green domain) to a strange one (yellow, red, and deep blue domains). In Fig. 4 the corresponding phase portraits (numerical iterations of a single initial condition) are shown for $M_{2}=0.85$ and $M_{1}$ varying along the dotted line in Fig. 3. Note that we do not have here a perioddoubling cascade like we would in a strongly dissipative case. Instead the transition to chaos follows a scenario from [92]): the orbit of period 2 (Fig. 4(b)) gives rise to a stable invariant pair of closed curves (Fig. 4(c)), which then "collide" to the saddle fixed point, and a Lorenz-like attractor is formed (Figs. 4(d,e)). This scenario is similar to the route to Lorenz attractor in the Morioka-Shimizu system [21-23]; one should just replace the symmetric equilibrium of the Morioka-Shimizu model by the saddle fixed point of our 3D-Henon map, the pair of asymmetric equilibria is replaced by the period-2 orbit, limit cycles of the Morioka-Shimizu model are replaced by closed invariant curves of the map. Obviously, this similarity must break at finer scales, e.g. instantaneous bifurcations of homoclinic loops in the model flow are replaced by continuous intervals of the existence of corresponding homoclinic orbits in the map, and a closed invariant curve of the map cannot just terminate at a homoclinic (like a limit cycle of the flow does), it must first lose smoothness and get destroyed [90].

As $M_{1}$ grows, the attractor grows in size and evolves into a strange attractor "without holes" (Figs. 4(h,i)). It reminds the attractor of Lorenz model after the absorbtion of the saddle-foci. This effect is related to a creation of a heterodimensional cycle where the one-dimensional unstable manifold 


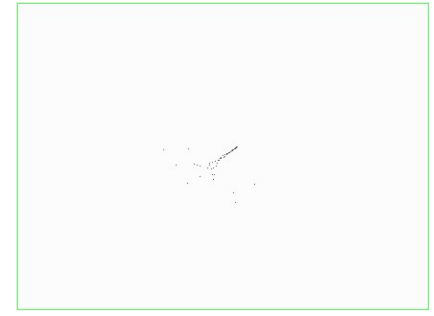

(a)

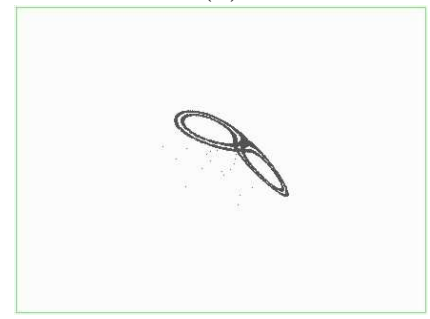

(d)

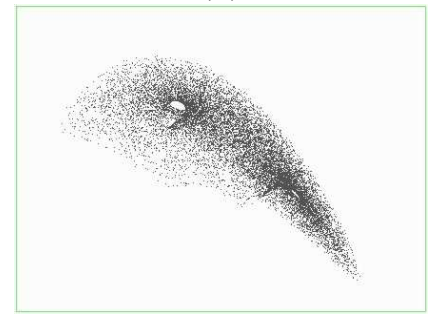

(g)

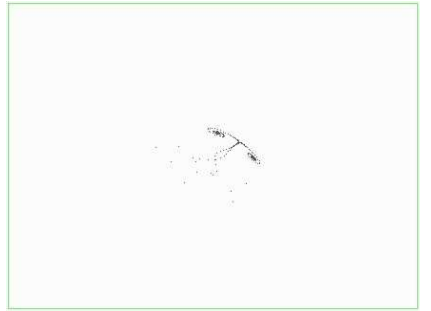

(b)

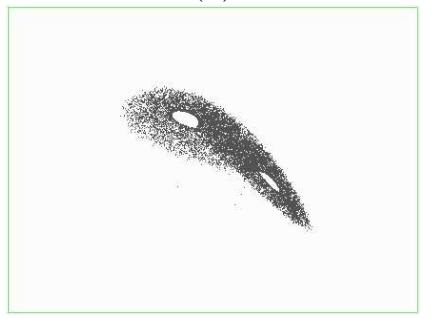

(e)

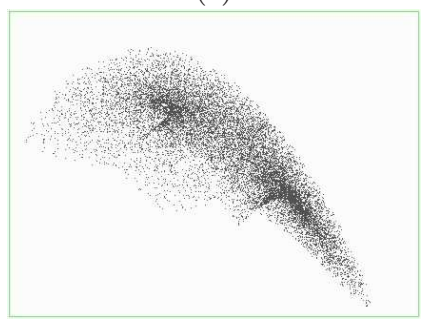

(h)

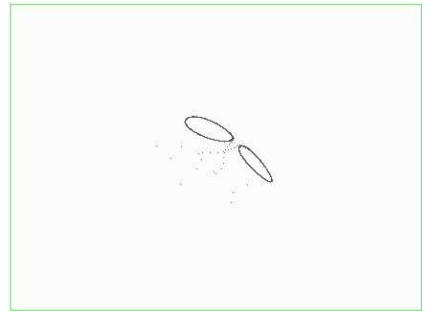

(c)

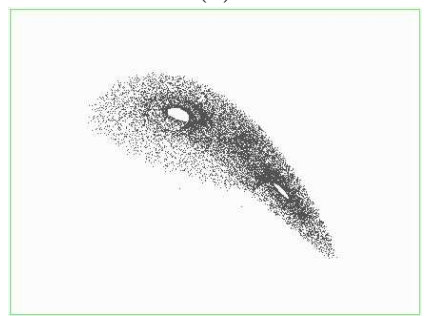

(f)

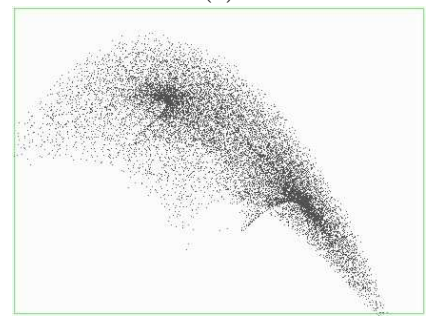

(i)

FiguRE 4. Evolution of attractors in the 3D Hénon map (3.11) with $B=0.7, M_{2}=0.85$, as $M_{1}$ varies: (a) $M_{1}=-0.055$ (fixed point); (b) $M_{1}=-0.045$ (period 2 point); (c) $M_{1}=-0.0342$ (closed invariant curve of period 2); (d) $M_{1}=-0.028$, (e) $M_{1}=0$, (f) $M_{1}=0.025$, (g) $M_{1}=0.05$, (h) $M_{1}=0.075$, (i) $M_{1}=0.1-$ strange attractors. All the figures are in the same scale.

of the saddle fixed point intersects with the one-dimensional stable manifold of the saddle-focus orbit of period two, analogous to the "Bykov contour" of the Lorenz model [131-133]. This bifurcation (e.g. in the Lorenz model [134-136] and in the Shimizu-Morioka model [21-23]) leads to creation of stable periodic orbits, i.e. the "no hole" attractor loses the pseudohyperbolic structure and becomes a quasiattractor in terms of [9], an attracting set of a non-trivial structure that may contain stable periodic orbits (or such orbits can be born at small perturbations of the system).

Next, we show results of numerical simulations of map (3.12). We start with the case $b=-3, d=$ $-3, a=1, M_{0}=-2$. At $M_{1}=4, B=1, A=0$ this map has a fixed point $($ at $x=y=z=1$ ) with the multipliers of $(-1,-1,1)$. It is easy to check that conditions (3.14) and (3.15) are satisfied, so we can expect the Lorenz-like attractor for parameter values close to these. We choose $B=0.7, A=0.1$, and varied $M_{1}$ from 4.365 down to 4.235. The results are shown in Fig. 5. The attractor forms in the way similar to the previous case: a cycle of period 2 bifurcates from a fixed point, then Andronov-Hopf bifurcation of the period-2 orbit creates a closed invariant curve of period 2, then the two components of this curve collide with the fixed point to eventually form a Lorenz-like attractor. The destruction of the attractor (Figs. 5(e-i)) proceeds via formation of a lacuna where a stable closed invariant curve emerges (Fig. 5(f)). Apparently, while the Lorenz-like attractor is no longer an attracting set, a large Shilnikov set (see Definition 2.6) of the saddle invariant tori that used to be a part of the attractor survives. This set seems to be a part of a new strange (quasi)attractor that no longer contains the saddle fixed 


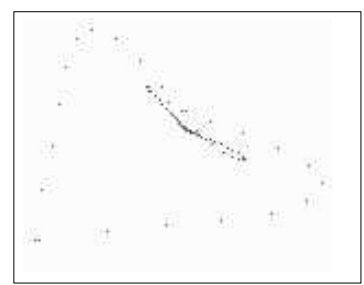

(a)

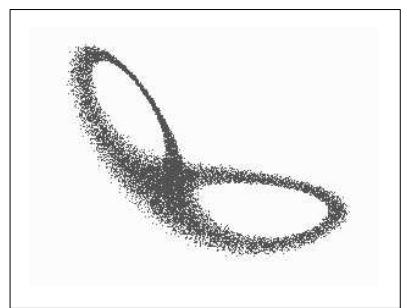

(d)

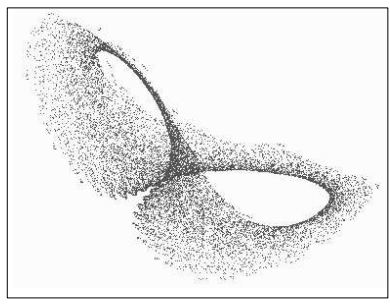

(g)

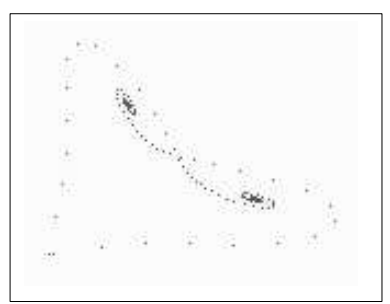

(b)

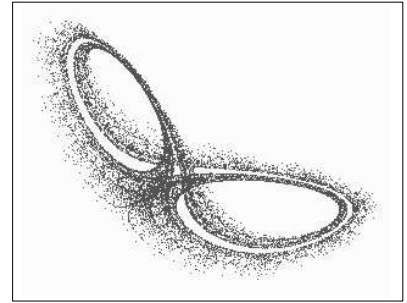

(e)

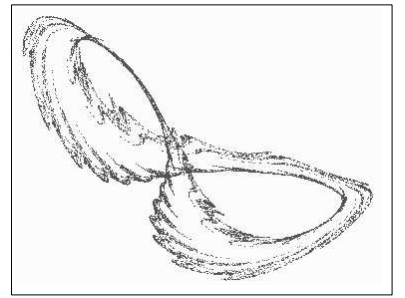

(h)

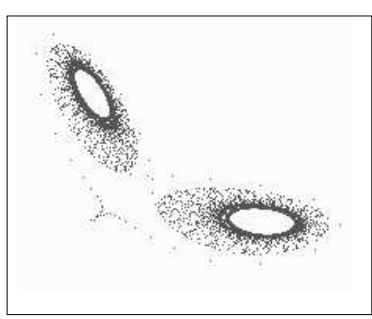

(c)

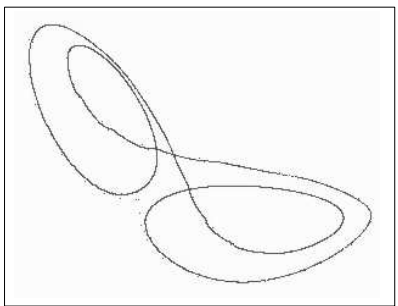

(f)

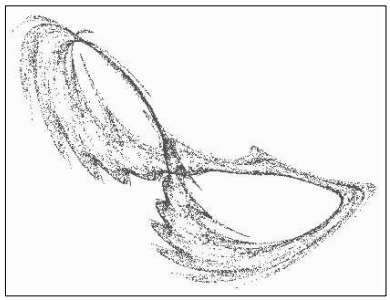

(i)

FiguRE 5. Creation and destruction of the Lorenz-like attractor in map (3.12) for $b=-3, d=$ $-3, c=1, M_{0}=-2, B=0.7, A=0.1$ as $M_{1}$ varies from 4.365 down to 4.235 . (a) stable fixed point; (b) cycle of period 2 (after period doubling); (c) closed invariant curve of period 2 (after AndronovHopf bifurcation of the cycle); (d) Lorenz-like attractor; (e) Lorenz-like attractor with a lacuna; (f)-(i) bifurcation stages after the destruction of the attractor (stable invariant curve, a quasiattractor that possibly contains a Shilnikov set of saddle invariant tori, and torus-chaos).

point (Fig. $5(\mathrm{~g}))$. The further decrease of $M_{2}$ creates a strange attractor (Figs. 5(h,i)) of the "wriggled" shape typical for the "torus-chaos" quasiattractor [86,88-90]. Note that the route of the destruction of the Lorenz-like attractor via formation of a lacuna, which we see in these figures, reminds one of the scenarios of the disappearance of the Lorenz attractor that was described in [3] and was also discovered in the Shimizu-Morioka model [21-23].

Another case corresponds to $b=2, d=1 / 3, c=0$. A fixed point at $x=y=z=1$ has multipliers $(-1,-1,1)$ at $M_{0}=-2, M_{1}=-2, B=1, A=-3$. Again, conditions (3.14) and (3.15) are satisfied. Numerics was performed at $M_{0}=3.67, B=0.7, C=-3.1$, with $M_{1}$ varying from -2.555 to -2.501 . The results are shown in Fig. 6. The first stages (Figs. 6(a-c)) on the route to the Lorenz-like attractor (Fig. 6(d)) are the same here as in the previous cases. The destruction of the attractor proceeds via formation of a lacuna where a stable invariant curve emerges (Fig. 6(e)), which then gives place to a strange quasiattractor (Fig. 6(f)). These stages are also similar to what is seen in Fig. 5. However, the quasiattractor has now a different structure, and unravels via a backward cascade of torus-doubling bifurcations (Figs. 6(f-i)). The last invariant curve disappears by colliding with a saddle invariant curve at a saddle-node bifurcation at $M_{1} \sim-2.501$. See [137-140] for the theory of the saddle-node and dou- 


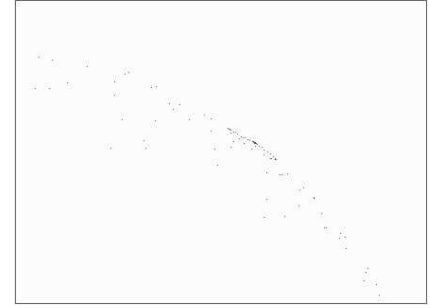

(a)

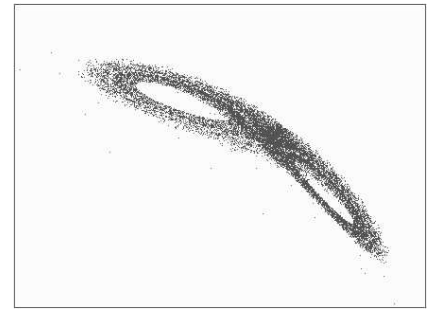

(d)

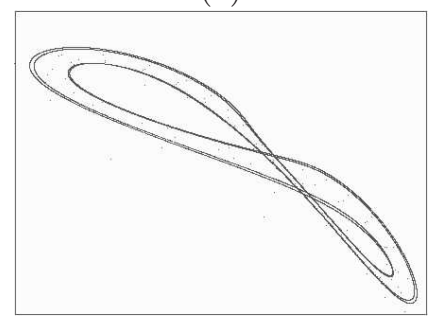

(g)

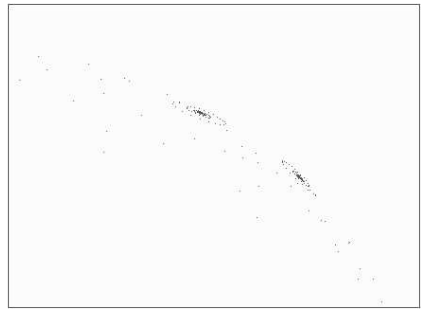

(b)

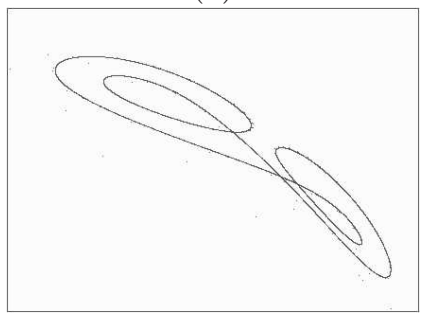

(e)

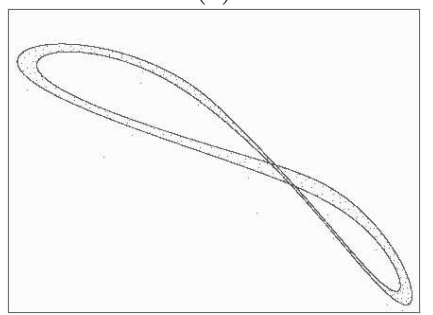

(h)

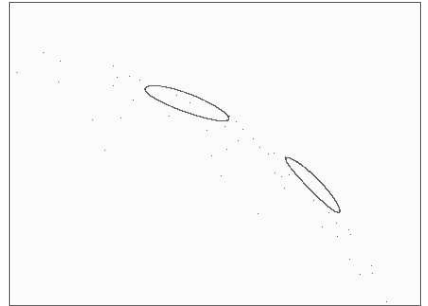

(c)

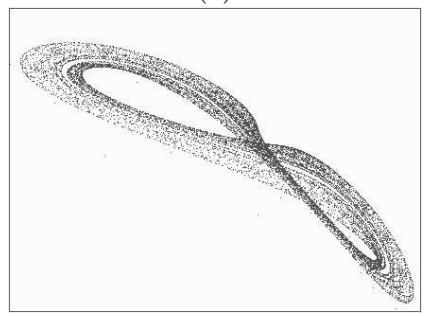

(f)

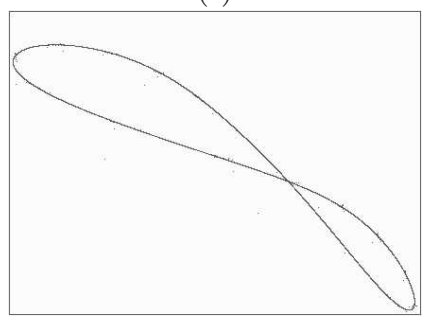

(i)

Figure 6. Plots of attractors of map (3.12) for $b=2, d=1 / 3, c=0, M_{0}=3.67, B=0.7, A=-3.1$ as $M_{1}$ varies from -2.555 to -2.505 . (a)-(d) as in Fig. 5 ; (e),(g),(h),(i) - stable closed invariant curves; (f) strange quasiattractor. All the figures are in the same scale

bling bifurcations for invariant curves. See numerical/experimental examples and theoretical discussions concerning cascades of torus-doublings in [141-145].

It is curious that, despite of our numerics being performed for parameter values sufficiently far from the bifurcation of a fixed point with the multipliers $(-1,-1,1)$, the bifurcations scenarios are quite similar to those one should have in its normal form, i.e. in the Shimizu-Morioka model with a small periodic forcing. Namely, the Lorenz attractor in Shimizu-Morioka model transforms into a strange quasiattractor in a variety of ways [21-23], depending on the choice of a path in the parameter plane, and this variety does include an absorption of saddle-foci like in Fig. 4, or formation of a lacuna with a consequent boundary crisis of the Lorenz attractor and emergence of a quasiattractor which may be accompanied by perioddoubling cascades or not like in Figs. 5,6. The differences between the destruction of the Lorenz-like attractor in our maps and the destruction of the Lorenz attractor in the model flow are still visible (mainly due to the effects of loss of smoothness and breakdown of invariant curves), but they do not seem to play a major role.

Acknowledgements. The paper has been supported by the grants of RFBR No.11-01-00001, No. 13-01-00589 and No.13-01-97028-povoljie, FCP "Cadry" No.14.B37.21.0361, No.14.B37.21.0862 and No.14.B37.21.0863, the Leverhulme Trust grant RPG-279, and a Royal Society grant. 


\section{References}

[1] S.V. Gonchenko, I.I. Ovsyannikov, C. Simó, D. Turaev. Three-dimensional Hénon-like maps and wild Lorenz-like attractors. Bifurcation and Chaos, 15 (2005), 3493-3508.

[2] V.S. Afraimovich, V.V. Bykov, L.P. Shilnikov. The origin and structure of the Lorenz attractor. Sov. Phys. Dokl., 22 (1977), 253-255.

[3] V.S. Afraimovich, V.V. Bykov, L.P. Shilnikov. On attracting structurally unstable limit sets of lorenz attractor type. Trans. Mosc. Math. Soc., 44 (1982), 153-216.

[4] J. Guckenheimer. A strange, strange attractor. In The Hopf Bifurcation Theorem and its Applications, eds. J. Marsden and M. McCracken, Springer-Verlag, 1976, 368-381.

[5] J. Guckenheimer, R.F. Williams. Structural stability of Lorenz attractors. IHES Publ. Math., 50 (1979), 59-72.

[6] R.F. Williams. The structure of Lorenz attractors. IHES Publ. Math., 50 (1979), 73-79.

[7] L.A. Bunimovich, Ya.G. Sinai. Stochasticity of the attractor in the Lorenz model. In Nonlinear Waves (Proc. Winter School, Moscow), Nauka, 1980, 212-226.

[8] C. Robinson. Differentiability of the stable foliation for the model Lorenz equations. Lect. Notes Math., 898 (1981), $302-315$.

[9] V.S. Aframovich, L.P. Shilnikov. Strange attractors and quasiattractors. in Nonlinear Dynamics and Turbulence, eds. G.I.Barenblatt, G.Iooss, D.D.Joseph, Boston, Pitmen, 1983.

[10] S.E. Newhouse. Non-density of Axiom A(a) on $S^{2}$. Proc. A.M.S. Symp. in Pure Math., 1 (1970), $191-202$.

[11] S.E. Newhouse. Diffeomorphisms with infinitely many sinks. Topology, 13 (1974), 9-18.

[12] S.E. Newhouse. The abundance of wild hyperbolic sets and non-smooth stable sets for diffeomorphisms. IHES Publ. Math., 50 (1979), 101-151.

[13] M.Benedicks, L.Carleson. The dynamics of the Henon map. Ann. Math., 133 (1991), 73-169.

[14] L. Mora, M. Viana. Abundance of strange attractors. Acta Math., 171 (1993), 1-71.

[15] Q.D. Wang, L.-S. Young. Strange attractors with one direction of instability. Commun. Math. Phys., 218 (2001), 1-97.

[16] R. Ures. On the Approximation of Hénon-like Attractors by Homoclinic Tangencies. Ergod. Th. Dyn. Sys. 15(1995), 1223-1229.

[17] A.S. Gonchenko, S.V. Gonchenko. On existence of Lorenz-like attractors in a nonholonomic model of celtic stones. Rus. J. Nonlin. Dyn., 9 (2013), 77-89.

[18] D.V. Turaev, L.P. Shilnikov. An example of a wild strange attractor. Sb. Math., 189 (1998), $291-314$.

[19] D.V. Turaev, L.P. Shilnikov. Pseudo-hyperbolisity and the problem on periodic perturbations of Lorenz-like attractors. Russian Dokl. Math., 467 (2008), 23-27.

[20] T. Shimizu, N. Morioka. On the bifurcation of a symmetric limit cycle to an asymmetric one in a simple model. Phys. Lett. A, 76 (1980), 201-204.

[21] A.L. Shilnikov. Bifurcation and chaos in the Morioka-Shimizu system. Methods of qualitative theory of differential equations, Gorky, 1986, 180-193 [English translation in Selecta Math. Soviet., 10 (1991) 105-117]; II. Methods of Qualitative Theory and Theory of Bifurcations, Gorky, 1989, 130-138.

[22] A.L. Shilnikov. On bifurcations of the Lorenz attractor in the Shimuizu-Morioka model. Physica D, 62 (1993), $338-346$.

[23] A.L. Shilnikov, L.P. Shilnikov, D.V. Turaev. Normal forms and Lorenz attractors. Bifurcation and Chaos, 3 (1993), 1123-1139.

[24] H.W.E. Jung. Uber ganze birationale Transformationen der Ebene. J. Reine Angew. Math., 184 (1942), $161-174$.

[25] S. Friedland, J. Milnor. Dynamical properties of plane polynomial automorphisms. Ergod. Th. Dyn. Sys., 9 (1989), 67-99.

[26] A.J. Dragt, D.T. Abell. Symplectic maps and computation of orbits in particle accelerators. In Integration algorithms and classical mechanics (Toronto, ON, 1993), Amer. Math. Soc., Providence, RI, 1996, 59-85.

[27] H.E. Lomeli, J.D. Meiss. Quadratic volume preserving maps. Nonlinearity, 11 (1998), 557-574.

[28] S.V. Gonchenko, V.S. Gonchenko, L.P. Shilnikov. On a homoclinic origin of Henon-like maps. Regul. Chaotic Dyn., 15 (2010), 462-481.

[29] L. Tedeschini-Lalli, J.A. Yorke. How often do simple dynamical processes have infinitely many coexisting sinks? Commun. Math. Phys., 106 (1986), 635-657.

[30] M. Henon. A two-dimensional mapping with a strange attractor. Commun. Math. Phys., 50 (1976), 69-77.

[31] N.K. Gavrilov, L.P. Shilnikov. On three-dimensional dynamical systems close to systems with a structurally unstable homoclinic curve. I. Math. USSR Sbornik, 17 (1972), 467-485; II. Math. USSR Sbornik, 19 (1973), $139-156$.

[32] S.V. Gonchenko, D.V. Turaev, L.P. Shilnikov. On models with a structurally unstable Poincare homoclinic curve. Sov. Math., Dokl., 44 (1992), 422-426.

[33] S.V.Gonchenko, L.P.Shilnikov, D.V.Turaev.On models with non-rough Poincare homoclinic curves. Physica D, 62 (1993), 1-14.

[34] S.V. Gonchenko, D.V. Turaev, L.P. Shilnikov. Dynamical phenomena in multi-dimensional systems with a non-rough Poincare homoclinic curve. Russ. Acad. Sci. Dokl. Math., 47 (1993), 410-415.

[35] S.V. Gonchenko, D.V. Turaev, L.P. Shilnikov. On the existence of Newhouse regions near systems with non-rough Poincare homoclinic curve (multidimensional case). Russian Acad. Sci.Dokl.Math. 47 (1993), 268-283.

[36] J. Palis, M. Viana. High dimension diffeomorphisms displaying infinitely many sinks. Ann. Math., 140 (1994), 91-136.

[37] N. Romero. Persistence of homoclinic tangencies in higher dimensions. Ergod. Th. Dyn. Sys., 15(1995), 735-757. 
[38] E. Colli. Infinitely many coexisting strange attractors. Ann. IHP, Anal. Non Lineaire, 15 (1998), 539-579.

[39] J.C. Tatjer. Three dimensional dissipative diffeomorphisms with homoclinic tangencies. Ergod. Th. Dyn. Systems, 21 (2001), 249-302.

[40] A.R. Champneys, J. Haerterich, B. Sandstede. A non-transverse homoclinic orbit to a saddle-node equilibrium. Ergod. Th. Dyn. Sys., 16 (1996), 431-450.

[41] D.V. Turaev D.V. On dimension of non-local bifurcational problems. Bifurcation and Chaos, 6 (1996), 919-948.

[42] S.V. Gonchenko, L.P. Shilnikov, D.V. Turaev. Dynamical phenomena in systems with structurally unstable Poincare homoclinic orbits. Chaos, 6 (1996), 15-31.

[43] S.V. Gonchenko, L.P. Shilnikov, D.V. Turaev. On Newhouse regions of two-dimensional diffeomorphisms close to a diffeomorphism with a nontransversal heteroclinic cycle. Proc. Steklov Inst. Math., 216 (1997), 70-118.

[44] S.V.Gonchenko, D.V.Turaev, L.P.Shilnikov. Quasiattractors and homoclinic tangencies. Comput. Math. Appl., 34 (1997), 195-227.

[45] S.V.Gonchenko, D.V.Turaev, L.P.Shilnikov. Homoclinic tangencies of an arbitrary order in Newhouse domains. In Itogi Nauki Tekh., Ser. Sovrem. Mat. Prilozh., 67 (1999), 69-128 [English translation in J. Math. Sci., 105 (2001), 1738-1778].

[46] S.V. Gonchenko, L.P. Shilnikov, D.V. Turaev. On dynamical properties of diffeomorphisms with homoclinic tangencies. Contemprary Mathematics and Its Applications, 7 (2003), 92-118 [English translation in J. Math. Sci., 126 (2005), 1317-1343].

[47] S.Gonchenko, L.Shilnikov, D.Turaev. Homoclinic tangencies of arbitrarily high orders in conservative and dissipative two-dimensional maps. Nonlinearity, 20 (2007), 241-275.

[48] S.V. Gonchenko, L.P. Shilnikov, D.V. Turaev. On dynamical properties of multidimensional diffeomorphisms from Newhouse regions. I. Nonlinearity, 21 (2008), 923-972.

[49] S.V. Gonchenko, L.P. Shilnikov, D.V. Turaev. On dynamical properties of multidimensional diffeomorphisms from Newhouse regions. II. submitted to Nonlinearity (2013).

[50] S.V.Gonchenko, I.I.Ovsyannikov, D.Turaev. On the effect of invisibility of stable periodic orbits at homoclinic bifurcations. Physica D, 241 (2012), 1115-1122.

[51] S.V.Gonchenko, V.S.Gonchenko. On Andronov-Hopf bifurcations of two-dimensional diffeomorphisms with homoclinic tangencies. preprint WIAS No.556, Berlin, 2000.

[52] S.V. Gonchenko, V.S. Gonchenko. On bifurcations of the birth of closed invariant curves in the case of two-dimensional diffeomorphisms with homoclinic tangencies. Proc. Steklov Inst. Math., 244 (2004), 80-105.

[53] V.S. Gonchenko, Yu.A. Kuznetsov, H.G.E. Meijer. Generalized Henon map and bifurcations of homoclinic tangencies. SIAM J. Appl. Dyn. Sys., 4 (2005), 407-436

[54] S.V. Gonchenko, O.V. Sten'kin, L.P. Shilnikov. On the existence of infinitely many stable and unstable invariant tori for systems from Newhouse regions with heteroclinic tangencies. Rus. J. Nonlinear Dynamics, 2 (2006), 3-25

[55] S.V. Gonchenko, V.S. Gonchenko, J.C. Tatjer. Bifurcations of three-dimensional diffeomorphisms with non-simple quadratic homoclinic tangencies and generalized Hénon maps. Regul. Chaotic Dyn., 12 (2007), 233-266.

[56] V.S. Gonchenko, L.P. Shilnikov. On bifurcations of systems with homoclinic loops to a saddle-focus with saddle index 1/2. Russian Dokl. Math., 76 (2007), 929-933.

[57] A.Gonchenko, S.Gonchenko. Towards a classification of linear and nonlinear Smale horseshoes. Rus. Nonlinear Dynamics, 3 (2007), 423-433.

[58] S. Gonchenko, M.-C. Li, M. Malkin. Generalized Henon maps and Smale horseshoes of new types. Bifurcation and Chaos, 18 (2008), 3029-3052.

[59] A. Gonchenko, S. Gonchenko, M. Malkin. On classification of classical and half-orientable horseshoes in terms of border points. Rus. Nonlinear Dynamics, 6 (2010), 549-566.

[60] V.S. Biragov. Bifurcations in two-parameter family of conservative mappings that are close to the Hénon map. Methods of Qualitative Theory and Theory of Bifurcations, Gorky, 1987, 10-24 [English translation in Selecta Math. Sov., 9 (1990), 273-282].

[61] V.Biragov, L. Shilnikov. On the bifurcation of a saddle-focus separatrix Loop in a three-dimensional conservative dynamical system. Methods of Qualitative Theory and Theory of Bifurcations, Gorky, 1989, 25-34 [English translation in Selecta Math. Soviet., 11 (1992), 333-340].

[62] L. Mora, N. Romero. Moser's invariant curves and homoclinic bifurcations. Dyn. Sys. Appl., 6 (1997), $29-42$.

[63] P.Duarte. Abundance of elliptic isles at conservative bifurcations. Dynam. Stability Systems, 14 (1999), 339-356.

[64] P. Duarte. Persistent Homoclinic Tangencies for Conservative Maps near the Identity. Ergod. Th. Dyn. Sys., 20 (2000), 393-438.

[65] P. Duarte. Elliptic Isles in Families of Area-Preserving Maps. Ergod. Th. Dyn. Sys., 28 (2008), $1781-1813$.

[66] V.Rom-Kedar, D.Turaev. Big islands in dispersing billiard-like potentials. Physica D, 130 (1999), $187-210$.

[67] D.Turaev, V.Rom-Kedar. Soft billiards with corners. J. Stat. Phys., 112 (2003), 765-813.

[68] S.V. Gonchenko, L.P. Shilnikov. On two-dimensional analytic area-preserving diffeomorphisms with infinitely many stable elliptic periodic points. Regul. Chaotic Dyn., 2 (1997), 106-123

[69] S.V. Gonchenko, L.P. Shilnikov. On two-dimensional area-preserving diffeomorphisms with infinitely many elliptic islands. J. Stat. Phys., 101 (2000), 321-356

[70] S.V. Gonchenko, L.P. Shilnikov. On two-dimensional area-preserving maps with homoclinic tangencies that have infinitely many generic elliptic periodic points. Notes Sci. seminars PDMI, 300(2003) [English translation in J. Math. Sci. 128 (2), 2767-2773]. 
[71] M.S. Gonchenko. On the structure of 1:4 resonances in Hénon maps. Bifurcation and Chaos, 15 (2005), 3653-3660.

[72] M.S. Gonchenko, S.V. Gonchenko. On cascades of elliptic periodic points in two-dimensional symplectic maps with homoclinic tangencies. Regul. Chaotic Dyn., 14 (2009), 116-136

[73] A. Gorodetski, V. Kaloshin. Conservative homoclinic bifurcations and some applications. Proc. Steklov Inst. Math., 267 (2009), 76-90.

[74] J.S.W. Lamb, O.V. Sten'kin. Newhouse regions for reversible systems with infinitely many stable, unstable and elliptic periodic orbits. Nonlinearity, 17 (2004), 1217-1244.

[75] S.V.Gonchenko, L.P.Shilnikov, D.Turaev. Elliptic periodic orbits near a homoclinic tangency in four-dimensional symplectic maps and Hamiltonian systems with three degrees of freedom. Regul. Chaotic Dyn., 3 (1998), 3-26.

[76] S.V.Gonchenko, D.V.Turaev, L.P.Shilnikov. Infinitely many elliptic periodic orbits in four-dimensional symplectic diffeomorphisms with a homoclinic tangency. Proc. Steklov Inst. Math., 244 (2004), 106-131.

[77] S.V. Gonchenko, J.D. Meiss, I.I. Ovsyannikov. Chaotic dynamics of three-dimensional Hénon maps that originate from a homoclinic bifurcation. Regul. Chaotic Dyn., 11 (2006), 191-212.

[78] S.V.Gonchenko, L.Shilnikov, D.Turaev. On global bifurcations in three-dimensional diffeomorphisms leading to wild Lorenz-like attractors. Regul. Chaotic Dyn., 14 (2009), 137-147.

[79] S.V. Gonchenko, I.I. Ovsyannikov. On bifurcations of three-dimensional diffeomorphisms with a non-transversal heteroclinic cycle containing saddle-foci. Rus. J. Nonlinear Dynamics, 6 (2010), 61-77

[80] D. Turaev. Polynomial approximations of symplectic dynamics and richness of chaos in non-hyperbolic areapreserving maps. Nonlinearity, 16 (2003), 123-135.

[81] D.Turaev. Maps close to identity and universal maps in the Newhouse domain. preprint arXiv:1009.0858, 2010.

[82] A. Delshams, S.V. Gonchenko, V.S. Gonchenko, J.T. Lazaro, O. Sten'kin. Abundance of attracting, repelling and elliptic periodic orbits in two-dimensional reversible maps. Nonlinearity, 26 (2013), 1-33.

[83] Homoclinic Tangencies, eds. S.V.Gonchenko and L.P.Shilnikov, Regular and Chaotic Dynamics, Moscow-Izhevsk, 2007.

[84] L.P. Shilnikov, A.L. Shilnikov, D.V. Turaev, L.O. Chua. Methods of qualitative theory in nonlinear dynamics. Part I, World Scientific, 1998; Part II, World Scientific, 2001.

[85] V.S. Afraimovich, L.P. Shilnikov. On small periodic perturbations of autonomous systems. Soviet Math., Dokl., 15 (1974), 206-211.

[86] V.S. Afraimovich, L.P. Shilnikov. On some global bifurcations connected with the disappearance of saddle-node fixed point. Soviet Math., Dokl., 15 (1974), 1761-1765.

[87] V.S. Afraimovich, L.P. Shilnikov. The ring principle in problems of interaction between two self-oscillating systems. J. Appl. Math. Mech., 41 (1977), 632-641

[88] J.H. Curry, J.A. Yorke. A transition from Hopf bifurcation to chaos: computer experiments with maps in $R^{2}$. In The Structure of Attractors in Dynamical Systems, eds. J.C. Martin, N.G. Markley, W. Perrizo, Lecture Notes Math., 668, Springer, Berlin, 1978, 48-66.

[89] D.G. Aronson, M.A. Chory, R.P. Mcgehee, G.R. Hall. Bifurcation from an invariant circle for two-parameter families of maps of the plane. Commun. Math. Phys., 83 (1982), 303-354.

[90] V.S. Afraimovich, L.P. Shilnikov. On invariant two-dimensional tori, their breakdown and stochasticity. Methods of the Qualitative Theory of Differential Equations, Gorky, 1983, 3-26 [English translation in: Amer. Math. Soc. Transl., 149 (1991), 201-212].

[91] A.Shilnikov, L.Shilnikov, D.Turaev. On some mathematical topics in classical synchronization. Bifurcation and Chaos, 14 (2004), 2143-2160.

[92] A.S. Gonchenko, S.V. Gonchenko, L.P. Shilnikov. Towards scenarios of chaos appearance in three-dimensional maps. Rus. J. Nonlinear Dynamics, 8 (2012), 3-28.

[93] A.V. Borisov, A.Yu. Jalnine, S.P. Kuznetsov, I.R. Sataev, J.V. Sedova. Dynamical phenomena occurring due to phase volume compression in nonholonomic model of the rattleback. Regul. Chaotic Dyn., 17 (2012), 512-532.

[94] C. Bonatti. $C^{1}$-generic dynamics: tame and wild behaviour. Proceedings of the ICM, Beijing 2002, V. 3, $279-294$.

[95] C. Bonatti, L. Diaz, E.R. Pujals. A $C^{1}$-generic dichotomy for diffeomorphisms: Weak forms of hyperbolicity or infinitely many sinks or sources. Ann. Math., 158 (2003), 355-418.

[96] C. Bonatti, L. Diaz, M. Viana. Dynamics Beyond Uniform Hyperbolicity: A Global Geometric and Probabilistic Perspective. Encyclopaedia of Mathematical Sciences, V. 102, Springer, 2005.

[97] C. Morales, E.R. Pujals. Singular strange attractors on the boundary of Morse-Smale systems. Ann. Sci. Ecole Norm. Sup., 30 (1997), 693-717.

[98] V. Araujo, M.J. Pacifico. Three-Dimensional Flows. Springer, 2010.

[99] S. Luzzatto, I. Melbourne, F. Paccaut. The Lorenz attractor is mixing. Comm. Math. Phys., 260 (2005), 393-401.

[100] Ya. B. Pesin. Dynamical systems with generalized hyperbolic attractors: hyperbolic, ergodic and topological properties. Ergod. Th. Dyn. Sys., 12 (1992), 123-151.

[101] E.A. Sataev. Invariant measures for hyperbolic maps with singularities. Russ. Math. Surv., 47 (1992), $191-251$.

[102] V.S. Afraimovich, N.I. Chernov, E.A. Sataev. Statistical properties of 2-D generalized hyperbolic attractors. Chaos 5 (1995), 238-252.

[103] C. Bonatti, A. Pumarino, M.Viana. Lorenz attractors with arbitrary expanding dimension. C.R. Acad. Sci. Paris, 325-I (1997), 883-888.

[104] J. Auslander, P. Seibert. Prolongations and stability in dynamical systems. Ann. Inst. Forier., Genoble, 14 (1964), $237-268$. 
[105] J. Auslander, N.P. Bhatia, P. Seibert. Attractors in dynamical systems. Bol. Soc. Mat. Mex., 9 (1964), 55-66.

[106] J. Milnor. On the concept of attractor. Comm. Math. Phys., 99 (1985, 177-195; Correction and remarks. Comm. Math. Phys., 102 (1985), 517-519.

[107] A. Gorodetski, Yu. Ilyashenko. Minimal and strange attractors. Bifurcation and Chaos, 6 (1996), $1177-1183$.

[108] C. Robinson. What is a Chaotic Attractor? Qualitative Theory Dyn. Sys., 7 (2008), 227-236.

[109] C. Conley. Isolated Invariant Sets and the Morse Index. CBMS Regional Conference Series Math., 38 (1978), Am. Math. Soc., Providence RI.

[110] D. Ruelle. Small random perturbations of dynamical systems and the definition of attractors. Comm. Math. Phys., 82 (1981), 137-151.

[111] M. Hurley. Attractors: persistence and density of their basins. Trans. Amer. Math. Soc., 269 (1982), $247-271$.

[112] V.A. Dobrynsky, A.N. Sharkovsky. Genericity of the dynamical systems, almost all trajectories of which are stable underconstantly acting perturbations. Soviet. Math. Dokl., 211 (1973).

[113] E.A. Sataev. Non-existence of stable trajectories in non-autonomous perturbations of systems of Lorenz type. Sb. Math., 196 (2005), 561-594.

[114] R. Bamon, J. Kiwi, J. Rivera. Wild Lorenz like attractors. Preprint (2005), arXiv:math/0508045.

[115] L.J. Diaz, J. Rocha. Nonconnected heteroclinic cycles: bifurcation and stability. Nonlinearity, 5 (1992), $1315-1341$.

[116] L.J. Diaz. Persistence of cycles and nonhyperbolic dynamics at heteroclinic bifurcations. Nonlinearity, 8 (1995), 693-713.

[117] Ch. Bonatti, L.J. Diaz. Persistent nonhyperbolic transitive diffeomorphisms. Ann. Math., 143 (1996), $367-396$.

[118] C. Bonatti, L.J. Diaz, E. Pujals, J. Rocha. Robust transitivity and heterodimensional cycles. Asterisque, 286 (2003), $187-222$.

[119] Ch. Bonatti, L.J. Diaz. Robust heterodimensional cycles and $C^{1}$-generic dynamics. J. Inst. of Math. Jussieu, 7 (2008), 469-525.

[120] L.J. Diaz, A. Gorodetski. Non-hyperbolic invariant measures for non-hyprerbolic homoclinic classes. Ergod. Th. Dyn. Sys., 29 (2009), 479-513.

[121] L.P. Silnikov. Structure of the neighborhood of a homoclinic tube of an invariant torus. Sov. Math. Dokl., 9 (1968), $624-628$.

[122] V.S. Afrajmovich, L.P. Shil'nikov. On a bifurcation of codimension 1 leading to the appearance of a countable set of tori. Sov. Math. Dokl., 25 (1982), 101-105.

[123] A. Gorodetski, Yu. Ilyashenko. Some new robust properties of invariant sets and attractors of dynamical systems. Functional Analysis and Applications, 33 (1999), 16-30.

[124] A. Gorodetski, Yu. Ilyashenko. Some properties of skew products over the horseshoe and solenoid. Proc. Steklov Inst. Math., 231 (2000), 96-118.

[125] A. Gorodetski, Yu. Ilyashenko, V. Kleptsyn, M. Nalskij. Non-removable zero Lyapunov exponent. Functional Analysis and Applications, 39 (2005), 27-38.

[126] V.N. Pisarevsky, A. Shilnikov, D. Turaev. Asymptotic normal forms for equilibria with a triplet of zero characteristic exponents in systems with symmetry. Regul. Chaotic Dyn., 2 (1998), 19-27.

[127] L.P. Shilnikov. The theory of bifurcations and quasiattractors. Russ. Math. Survey, 36[4] (1981), $240-241$.

[128] C. Robinson. Homoclinic bifurcation to a transitive attractor of the Lorenz type. Nonlinearity, 2 (1989), 495-518; II. SIAM J. Math. Anal., 23 (1992), 1255-1268.

[129] M. Rychlic. Lorenz attractors through Shilnikov type bifurcation. Ergod. Th. Dyn. Sys., 10 (1990), $793-821$.

[130] G.Tigan, D.Turaev. Analytical search for homoclinic bifurcations in Morioka-Shimizu model. Physica D, 240 (2011), 985-989.

[131] V.V. Bykov. On the structure of a neighborhood of a separatrix contour with a saddle-focus. Methods of the Qualitative Theory of Differencial Equations, Gorky, 1978, 3-32.

[132] V.V. Bykov. On bifurcations of dynamical systems close to systems with a separatrix contour containing a saddlefocus. Methods of the Qualitative Theory of Differencial Equations, Gorky, 1980, 44-72.

[133] V.V. Bykov. The bifurcations of separatrix contours and chaos. Physica D, 62(1993), 290-299.

[134] N.V. Petrovskaya, V.I. Yudovich. Homoclinic loops of the Saltzman-Lorenz system. Methods of qualitative theory of differential equations, Gorky, 1980, 73-83.

[135] V.V. Bykov, A.L. Shilnikov. On the boundaries of the domain of existence of the Lorenz attractor. Methods of qualitative theory and bifurcation theory, Gorky, 1989, 151-159 [English translation in Selecta Math. Soviet., 11 (1992), 375-382].

[136] V.S. Afraimovich, V.V. Bykov, L.P. Shilnikov. On the existence of stable periodic orbits in the Lorenz model. Russ. Math. Survey, 35[4] (1980), 164-165.

[137] A. Chenciner. Bifurcations de points fixes elliptiques. I. Courbes invariantes. IHES Publ. Math., 61 (1985), 67-127; II. Orbites periodiques et ensembles de Cantor invariants. Invent. Math., 80 (1985), 81-106; III. Orbites periodiques de "petites" periodes et elimination resonante des couples de courbes invariantes. IHES Publ. Math., 66 (1987), 5-91.

[138] J.E. Los.Nonnormally hyperbolic invariant curves for maps in $R^{3}$ and doubling bifurcation. Nonlinearity, 2 (1989), 149-174.

[139] H.W. Broer, G.B. Huitema, F. Takens. Unfoldings of quasi-periodic tori. Mem. AMS, 83 (1990), 1-82.

[140] B.L.J. Braaksma, H.W. Broer, G.B. Huitema. Toward a quasi-periodic bifurcation theory. Mem. AMS, 83 (1990), 83-175. 
[141] A. Arneodo, P.H. Coullet, E.A. Spiegel. Cascade of period doublings of tori. Phys. Lett. A, 94 (1983), 1-5.

[142] V. Franceschini. Bifurcations of tori and phase locking in a dissipative system of differential equations. Physica D, 6 (1983), 285-304.

[143] V.S. Anishchenko, V.V. Astakhov, T.E. Letchford, M.A. Safonova. Structure of the quasihyperbolic stochasticity in an inertial self-excited oscillator. Radiophysics and Quantum Electronics, 26 (1983), 619-628.

[144] V.S. Anishenko. Complex oscillations in simple systems. Nauka, Moscow, 1990.

[145] K. Kaneko. Collapse of Tori and Genesis of Chaos in Dissipative Systems. World Scientific, Singapore, 1986.

[146] C. Bonatti, Y. Shi, Robustly transitive attractor derived from Lorenz, in preparation. 